\title{
Les premiers Grecs en France : le cas de Béziers I/ Rhòde
}

The First Greeks in France: The Case of Beziers I/Rhòde

\section{Élian Gomez et Daniela Ugolini}

\section{(2) OpenEdition}

1 Journals

Édition électronique

URL : https://journals.openedition.org/gaia/901

DOI : 10.4000/gaia.901

ISSN : 2275-4776

Éditeur

UGA Éditions/Université Grenoble Alpes

\section{Édition imprimée}

ISBN : 978-2-37747-199-7

ISSN : 1287-3349

\section{Référence électronique}

Élian Gomez et Daniela Ugolini, « Les premiers Grecs en France : le cas de Béziers I/Rhòde », Gaia [En ligne], 22-23 | 2020, mis en ligne le 30 juin 2020, consulté le 09 décembre 2021. URL : http:// journals.openedition.org/gaia/901; DOI : https://doi.org/10.4000/gaia.901

Ce document a été généré automatiquement le 9 décembre 2021.

Gaia. Revue interdisciplinaire sur la Grèce archaïque 


\section{Les premiers Grecs en France : le cas de Béziers I/Rhòde}

The First Greeks in France: The Case of Beziers I/Rhòde

Élian Gomez et Daniela Ugolini

\section{Introduction 1}

1 À l'échelle historiographique, Béziers I/Rhòde (fig. 1) a émergé récemment dans le cadre de la colonisation ou des diasporas grecques et, jointe à d'autres arguments, donne crédit à la priorité des Rhodiens (ou Doriens) - mentionnée par les sources - dans l'exploration du Nord-Ouest méditerranéen et leur consécutive implantation, que l'exégèse avait réduites à une légende sans valeur historique. 
Fig. 1. - Localisation de Béziers.

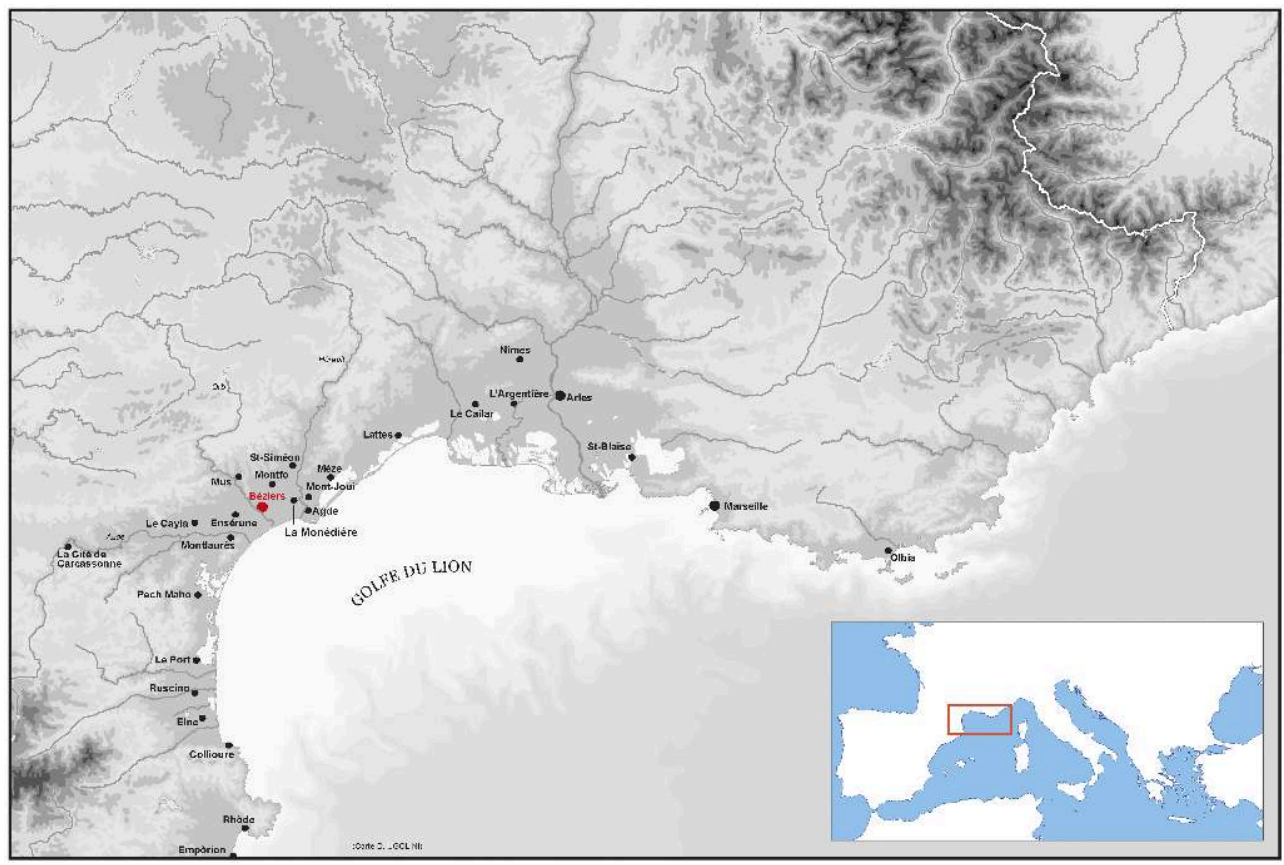

Carte de D. Ugolini.

2 L'occupation grecque - inattendue car dans un lieu que, de prime abord, rien ne désignait pour cela - a été identifiée lors de la première opération archéologique d'une certaine ampleur $(1985)^{2}$ et a suscité surprise et perplexité. Depuis, le dossier s'est considérablement enrichi, mais l'absence d'un nom proche de l'actuel parmi ceux des établissements grecs mentionnés par les textes anciens a représenté un obstacle à sa pleine reconnaissance. Grâce à diverses études et aux opérations préventives des dernières années ${ }^{3}$, la phase des premiers contacts entre Grecs et autochtones est mieux cernée, la chronologie des débuts de l'établissement grec est circonscrite, les aspects mobiliers sont en grande partie définis et les immobiliers en progrès, les interactions avec les sites de la région en partie appréhendées. La période archaïque étant maintenant documentée, un nom (Rhòde) - identifié de longue date dans les sources mais qu'il était risqué d'avancer avant de disposer d'un appui archéologique - a été proposé pour cette ville oubliée et longtemps anonyme.

évitablement, des zones d'ombre persistent. Par exemple, l'origine du premier groupe de Grecs ne peut être précisée et il y a fort à parier qu'elle était plurielle, comme souvent dans les entreprises coloniales ${ }^{4}$, mais la Sicile dorienne est tout particulièrement concernée par des relations avec notre côte qui pourraient bien correspondre à un " programme » d'expansion vers l'ouest. De plus, l'île de Rhodes et la Rhòde biterroise se trouvant aux deux extrémités de la Méditerranée dorienne avec la Sicile au milieu, il est tentant d'envisager que le nom de la colonie ${ }^{5}$ la plus occidentale ait été choisi pour son fort pouvoir évocateur, une vertu qu'il aura à nouveau lorsque sera fondée, probablement par des émigrés rhodo-biterrois, Rhòde d'Ibérie.

Insérer la ville dans le cadre historique en vigueur n'était pas simple, car la composante dorienne s'était plus ou moins perdue dans les méandres de l'histoire. Il a donc fallu le remodeler, car la Rhòde archaïque des sources retrouve toute son épaisseur si associée aux données biterroises ${ }^{6}$ et la date retenue pour les premiers grands travaux mis au 
jour à Saint-Jacques est 625-600 d'après le mobilier, et peut-être plutôt $625^{7}$. Toutefois, la fréquentation grecque peut être plus ancienne, comme le suggère un tesson grec bien daté vers 640 (fig. 2).

Fig. 2. - Saint-Jacques 2017-2018. Fragment de cruche de style protocorinthien polychrome (sicilienne ? de Grèce de l'Est ?), vers 640.

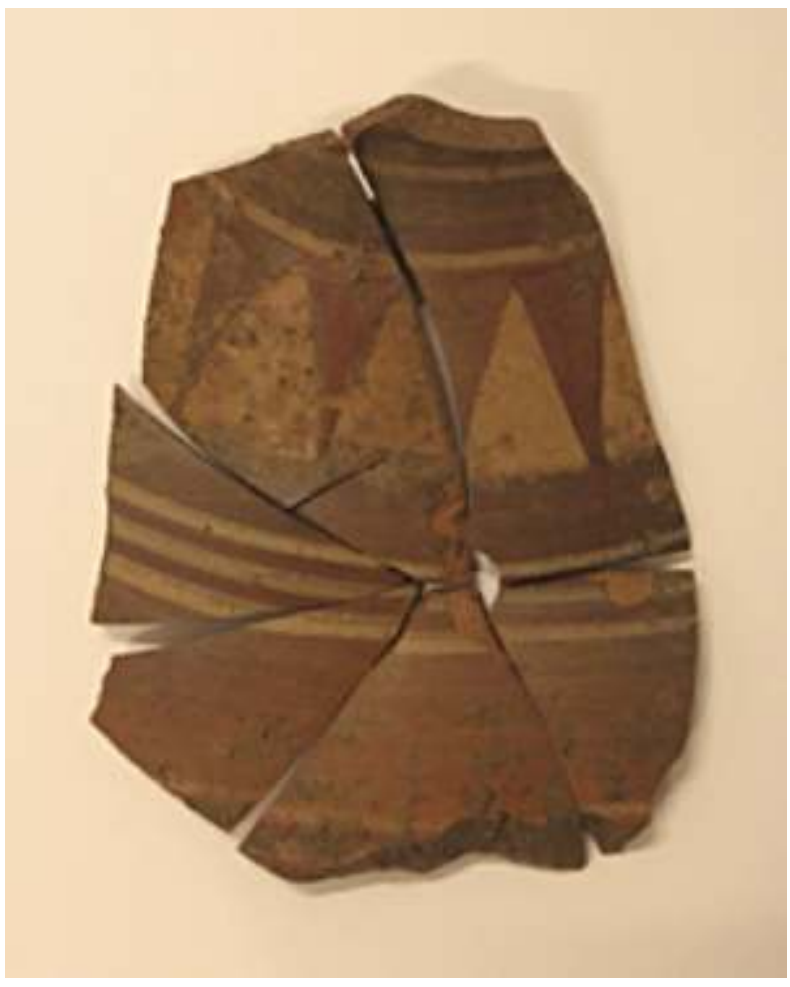

Cliché de É. Gomez.

$5 \quad$ Il est le plus ancien du Midi trouvé dans un contexte non funéraire mais contemporain des vases grecs des tombes indigènes entre l'Hérault et l'Aude. Il ne suffit pas à faire coïncider les débuts de l'établissement avec cette date, mais il est là et sa présence n'a pas de sens dans un lieu inhabité. Il peut s'agir d'un souvenir amené plus tard, mais il est plus vraisemblable que l'on ait commencé à visiter les deux collines biterroises à ce moment, et peut-être à s'y établir. En attendant que d'autres découvertes viennent préciser ce point, il faut tenir compte du fait que les niveaux de construction et de fonctionnement de la fortification de Saint-Jacques ont été emportés depuis l'Antiquité et que seules les structures creusées étaient partiellement préservées. Autrement dit, nous ignorons à partir de quelle altitude ont été excavés les fossés et, par conséquent, à quel moment précis du $\mathrm{VII}^{\mathrm{e}}$ siècle. Nous n'avons donc pas le mobilier de la toute première installation, mais celui gisant au fond des excavations, préservé en quelques points, qui correspond à l'abandon de la phase bâtie/excavée. Ainsi, nous nous autorisons à ne pas exclure une présence antérieure à 625, peut-être sous la forme d'un campement. Quoi qu'il en soit, l'écart chronologique entre, d'une part, Béziers I/Rhòde et le Biterrois (d'où proviennent tous les plus anciens vases grecs du Midi) et, d'autre part, Marseille/Massalia détache le mouvement doro-rhodien de celui ioniophocéen qui l'a suivi et concerné essentiellement la Provence.

6 Les fouilles étant rares dans une ville peu soumise aux réaménagements modernes, certains éléments constitutifs de la première Rhòde d'Occident manquent encore à 
l'appel ${ }^{8}$, mais d'autres illustrent les débuts et l'évolution d'une ville que nous considérons comme une pòlis.

\section{La position géographique}

7 Le lieu a été choisi pour ses innombrables atouts, qu'il est utile de rappeler tant cet aspect, pourtant majeur, est sous-estimé.

8 Béziers est à l'intérieur des terres (actuellement distante de 12-14 km de la mer) et suffisamment éloignée des marécages côtiers pour ne pas en subir les inconvénients. Elle occupe deux collines (Saint-Jacques et Saint-Nazaire) séparées par un thalweg au fond duquel passaient la mythique voie Héracléenne, puis la romaine voie Domitienne et c'est encore là que court la départementale D 613. Les deux collines offrent une vue dominante jusqu'aux Corbières et Pyrénées à l'ouest/sud-ouest, jusqu'à l'étang de Thau à l'est, entre la mer au sud et la Montagne Noire et les Cévennes au nord. Naturellement défendues par des falaises sur trois côtés, seul l'est présente une pente douce. Le fleuve Orb coule à leur pied du côté occidental (fig. 3) et c'est ici que le Lirou, dont la vallée est l'accès le plus direct à la région de Castres, y conflue. Deux sources pérennes (Puits de L'Inquant/Embroucadou et Grazilhan) sourdent sur la plus grande colline SaintNazaire, où a été principalement développée l'occupation. Une troisième s'écoulait aux abords orientaux de la ville (immédiatement au-delà des allées Paul-Riquet).

Fig. 3. - Vue de Béziers.

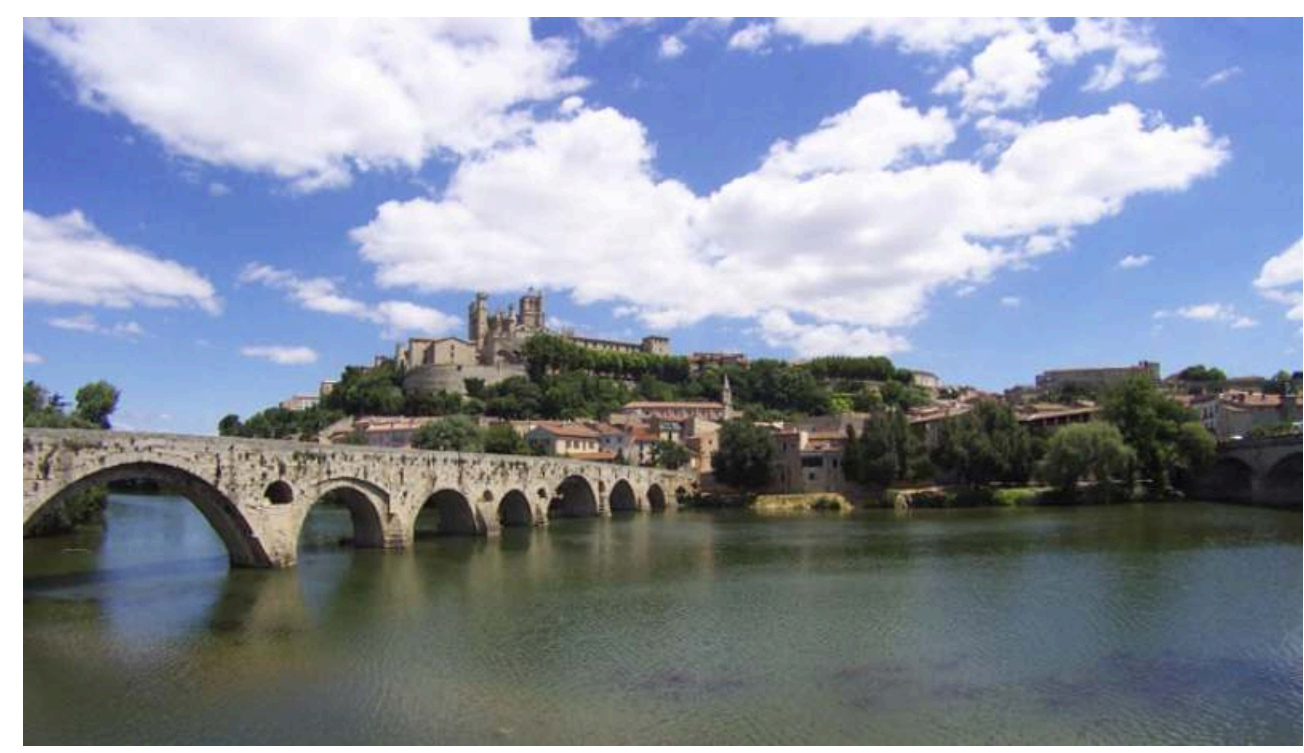

Cliché de C. Olive.

9 La ville est située au seul endroit où le franchissement de l'Orb est relativement facile, entre une plaine côtière marécageuse et changeante et un arrière-pays où le fleuve est très encaissé. C'est encore aujourd'hui un passage obligé de la circulation terrestre estouest (fig. 4) et un lieu central dans l'aire occidentale, entre les deux fleuves principaux que sont l'Hérault et l'Aude. 
Fig. 4. - Ponts sur l'Orb.

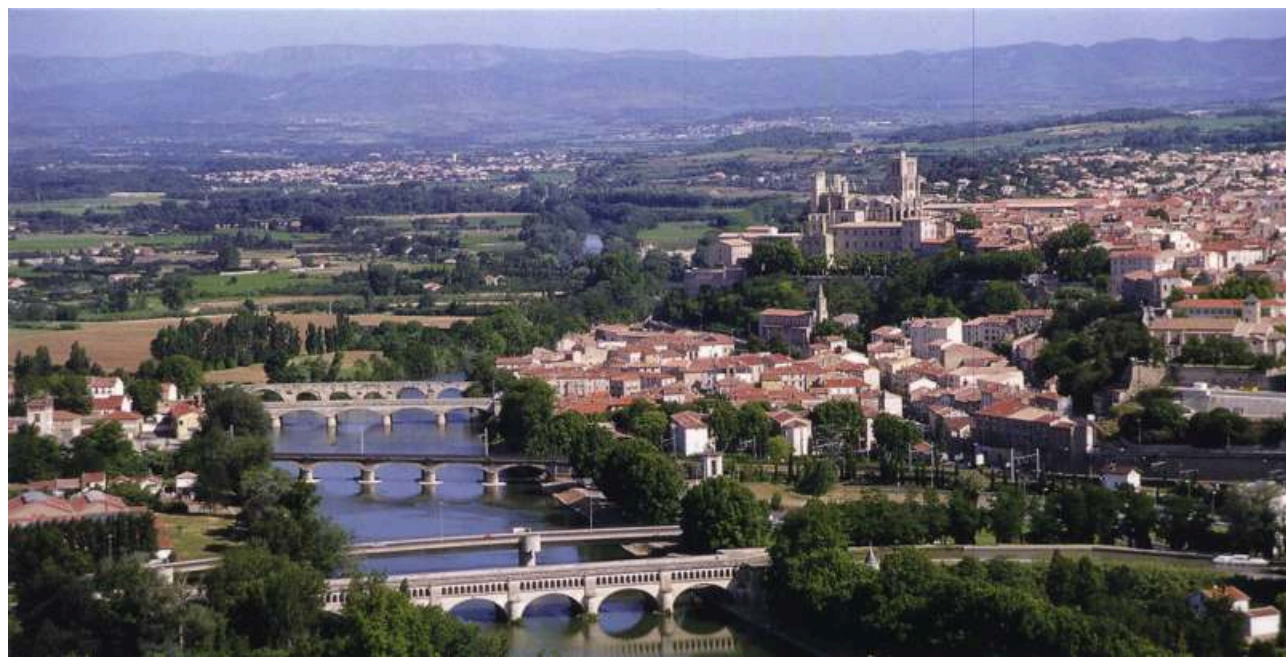

Cliché de C. Olive.

10 De Béziers on accède à toutes les vallées de cette zone, y compris à celles directes vers le Massif Central. Notons que la voie la plus facile vers l'Atlantique et le pied du Massif Central est celle au départ de Béziers en rive gauche de l'Aude puis en rive droite de la Garonne. La particulière configuration géographique de ce carrefour, qui a été longtemps au cœur de l'économie locale ${ }^{9}$, en a fait toute l'importance.

11 L'environnement est légèrement vallonné, fertile et sillonné par un important réseau de rivières et ruisseaux fournissant l'eau nécessaire à l'agriculture, l'élevage, l'artisanat. Des secteurs miniers sont connus dans l'arrière-pays (Cabrières, Monts d'Orb, Montagne Noire), mais leur exploitation - bien que mise en avant ${ }^{10}$ - n'est pas réellement prouvée pour la phase précoloniale et coloniale.

On ignore où se trouvait l'ancienne embouchure de l'orb tant la plaine deltaïque est instable, mais la navigation, au moins par de petits bateaux, ne devait pas poser problème et, au Moyen Âge, un port de haute mer a fonctionné à Sérignan. Pour ses liaisons maritimes, Béziers I/Rhòde a compté aussi depuis le début sur l'Hérault, plus important que l'orb, d'abord par l'intermédiaire du site indigène de Bessan/ La Monédière, puis par la création du port grec d'Agde I/Agàthe. La possibilité d'un autre port est actuellement envisagée à l'ouest, au bord de l'étang de Vendres, à environ $8 \mathrm{~km}$ de la ville, qui forme un golfe encore relativement profond, flanqué par le relief de La Clape faisant office d'amer. Le plan d'eau était sans doute favorable à cette fonction avant le changement du cours de l'Aude (au Moyen Âge), qui depuis y déverse ses sédiments et le comble. Des lignes cadastrales nord-sud dans le parcellaire actuel le relient à la ville. Déjà présentes dans celui d'époque romaine, elles reprennent peutêtre une division des terres de la phase grecque. Des sites ruraux de cette période sont d'ailleurs alignés entre Béziers et Vendres et la question aura un développement spécifique. 


\section{Installation et évolution}

\subsection{La première occupation sur la colline Saint-Jacques}

13 À l'heure actuelle, la plus ancienne occupation bien documentée est celle de la colline Saint-Jacques, un emplacement stratégique offrant une vue panoramique dans toutes les directions (fig. 5). Une puissante fortification y a été dressée au plus tard dans le dernier quart du viI ${ }^{\mathrm{e}}$ siècle $^{11}$.

Fig. 5. - Extension de la ville de Béziers I/Rhòde.
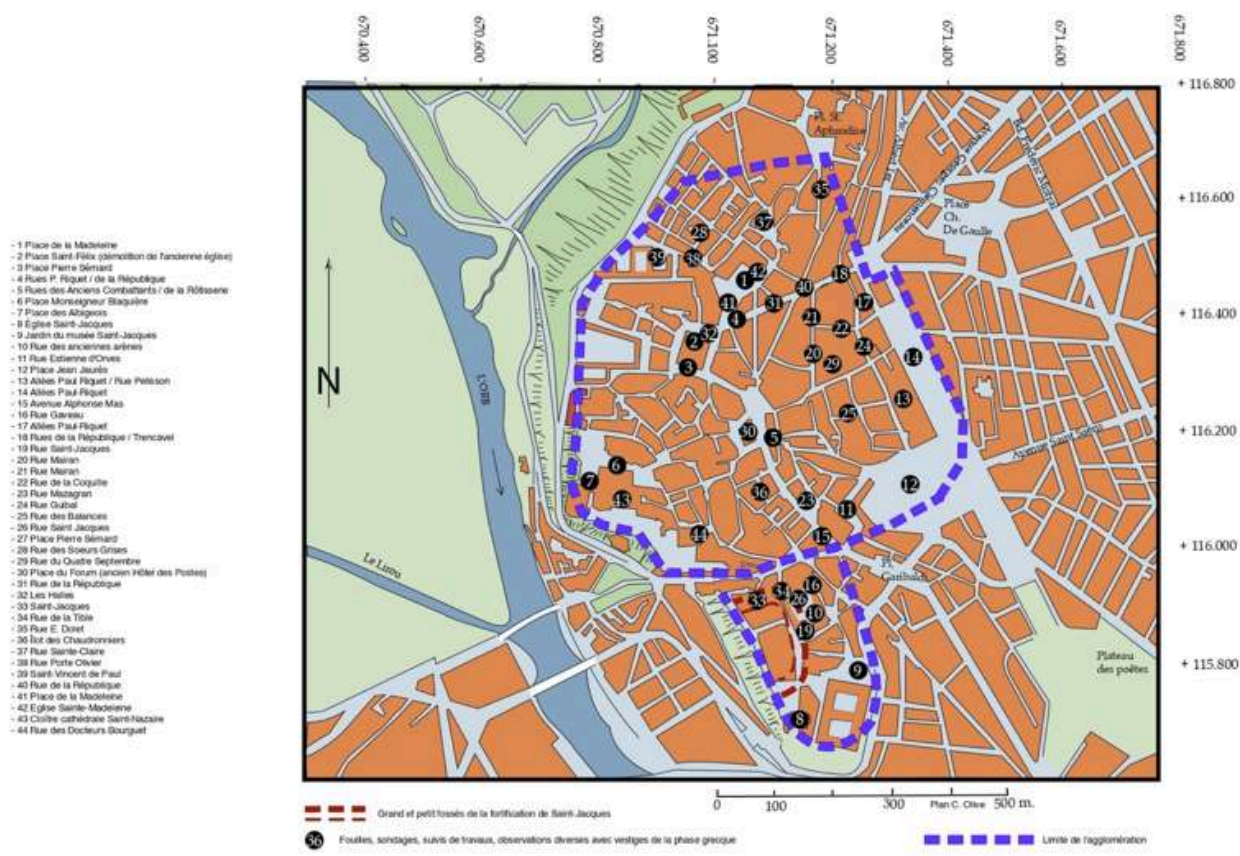

Le périmètre proposé (en violet) tient compte des opérations archéologiques à l'extérieur de celui-ci n'ayant livré aucune trace de la phase grecque.

Plan de C. Olive, modifié

Seules les structures excavées étaient conservées (fig. 6). 
Fig. 6. - Saint-Jacques 2017-2018. La fortification construite vers 625.

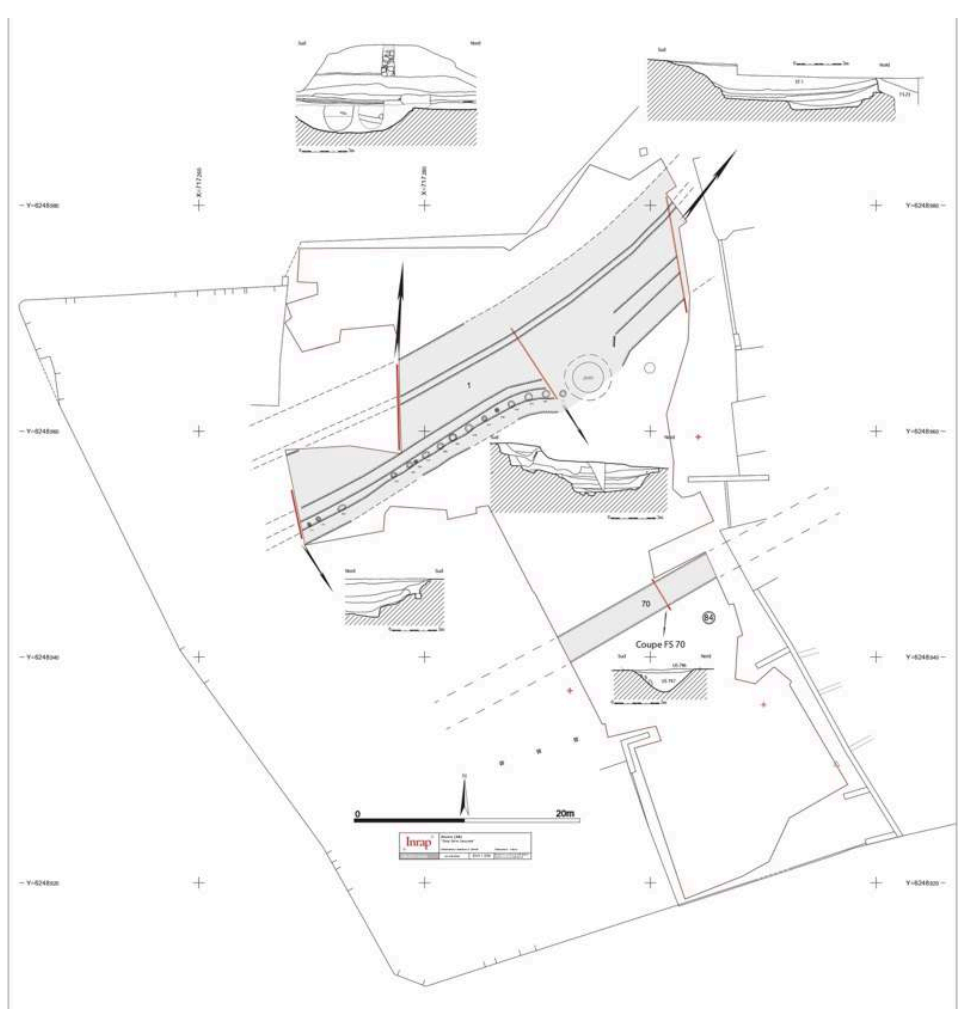

Plan et coupes de C. Bioul et É. Gomez.

15 - Un grand fossé (ST1), large de 10-12 m et d'une profondeur estimée d'au moins $4 \mathrm{~m}$, a été creusé en escalier.

16 - La terre de l'excavation a servi à constituer un agger, d'une hauteur estimée de 7-8 m au point le plus haut, retenu par une puissante palissade (trous de poteaux de 0,40 à $0,60 \mathrm{~m}$ de diamètre, régulièrement espacés) du côté interne du fossé et par une deuxième plus légère et sans doute plus basse installée dans un fossé étroit (ST70), parallèle au premier et à $20 \mathrm{~m}$ de distance.

17 - Une entrée en entonnoir était aménagée du côté longeant le thalweg séparant la colline de celle de Saint-Nazaire, où court la voie et à proximité immédiate du franchissement de l'Orb. Détruite par un très grand puits du $\mathrm{Xv}^{\mathrm{e}}$ siècle, à l'ouest elle était matérialisée par la déviation de l'alignement des trous de poteaux de la paroi interne du grand fossé et, à l'est (plus profondément arasé), par la direction de sa bordure. Le franchissement du fossé se faisait probablement sur une bande réservée : un pont en bois ? Cet accès se trouve dans l'un des seuls points où il était possible et utile et c'est là qu'il était idéalement prédisposé à la communication avec la colline voisine et à la surveillance de la route amenant au fleuve.

18 - À l'intérieur de la fortification, à côté de l'entrée, un grand creusement tronconique dans les marnes du substrat était une citerne complétée jusqu'à l'embouchure par un conduit cylindrique dont on aperçoit l'amorce ( $\varnothing$ conservé de l'ouverture : 0,80 $\mathrm{m} ; \varnothing \mathrm{du}$ fond: $2,20 \mathrm{~m}$; profondeur conservée : $2,40 \mathrm{~m}$; volume conservé : $\pm 4,5 / 5 \mathrm{~m}^{3}$ ) (fig. 7). Dans son comblement d'abandon, daté vers 575 , un abondant mobilier a été récupéré : des vases en majorité non tournés typiques d'une phase encore ancienne du premier âge du fer, des vases grecs de diverses origines et d'autres déjà de production locale, 
parmi lesquels d'évidents rebuts de cuisson de céramique grise monochrome et des fragments de fours de potier démolis.

Fig. 7. - Saint-Jacques 2017-2018. La citerne (ST84).

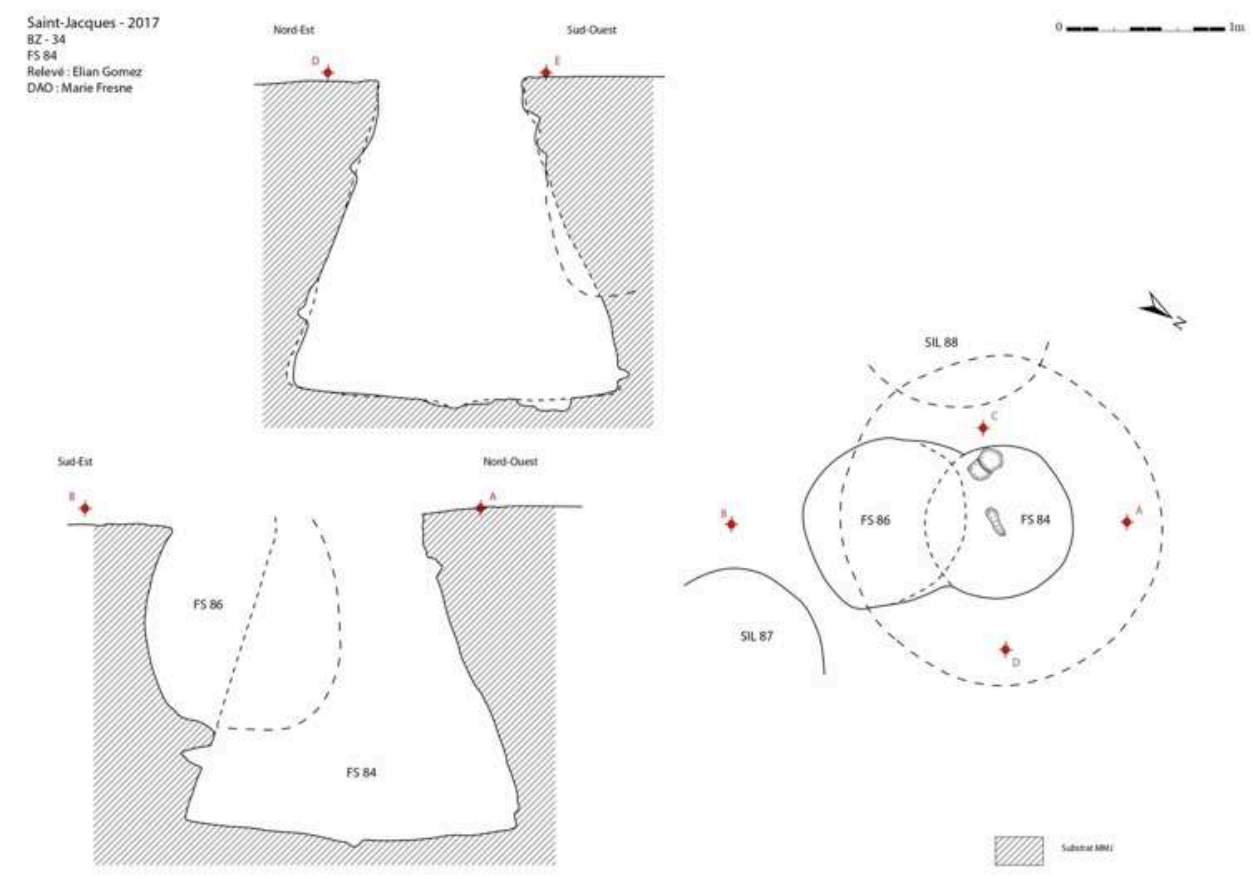

Relevé et DAO de É. Gomez et M. Fresne. il rejoignait la défense naturelle de la falaise occidentale surplombant l'orb et englobait environ 3-3,5 ha pour une superficie interne utile d'à peu près 1,5 ha (fig. $5, n^{\circ s} 33,34$, $26,19)$. Malgré l'absence d'une plateforme entre la palissade de l'escarpe et le fossé, le système défensif trouve des comparaisons convaincantes en Italie et en Sicile, notamment à Mégara Hyblaea dans le courant du vII ${ }^{\mathrm{e}}$ siècle, à Poseidonia et peut-être à Marseille au vi ${ }^{e}$ siècle $^{13}$.

Les niveaux d'usage n'étant pas conservés, on ignore comment était organisé ce lieu. L'importance de l'ouvrage défensif laisse supposer un climat de méfiance. Son abandon autour de $575^{14}$, prouve qu'il a été en fonction pendant un laps de temps relativement court. On en déduit, d'une part, qu'il a pu se révéler inutile dans un contexte humain environnant pacifique et/ou, d'autre part, que la colline voisine - plus étendue - a été préférée pour le développement urbain. Cette zone a toutefois continué à être occupée, notamment par des artisans.

\subsection{L'habitat se développe}

À partir des années autour de 600, l'habitat se développe sur la colline Saint-Nazaire et le $v^{e}$ siècle est celui de l'apogée, lorsque la ville couvre les deux collines pour une superficie densément occupée de 35-40 ha ${ }^{15}$ (fig. 5). Béziers I/Rhòde est alors, et de loin, le plus grand site à l'ouest du Rhône. 
Les vestiges attestent un plan orthogonal nord-sud en place dès au moins le deuxième quart/milieu $\mathrm{du} \mathrm{VI}^{\mathrm{e}}$ siècle. Un deuxième système, en biais de $30^{\circ}$ par rapport au précédent pour des raisons topographiques, concerne le quartier nord, implanté dans le deuxième quart du $\mathrm{v}^{\mathrm{e}}$ siècle (fig. 8).

Fig. 8. - Plan cadastral de Béziers.

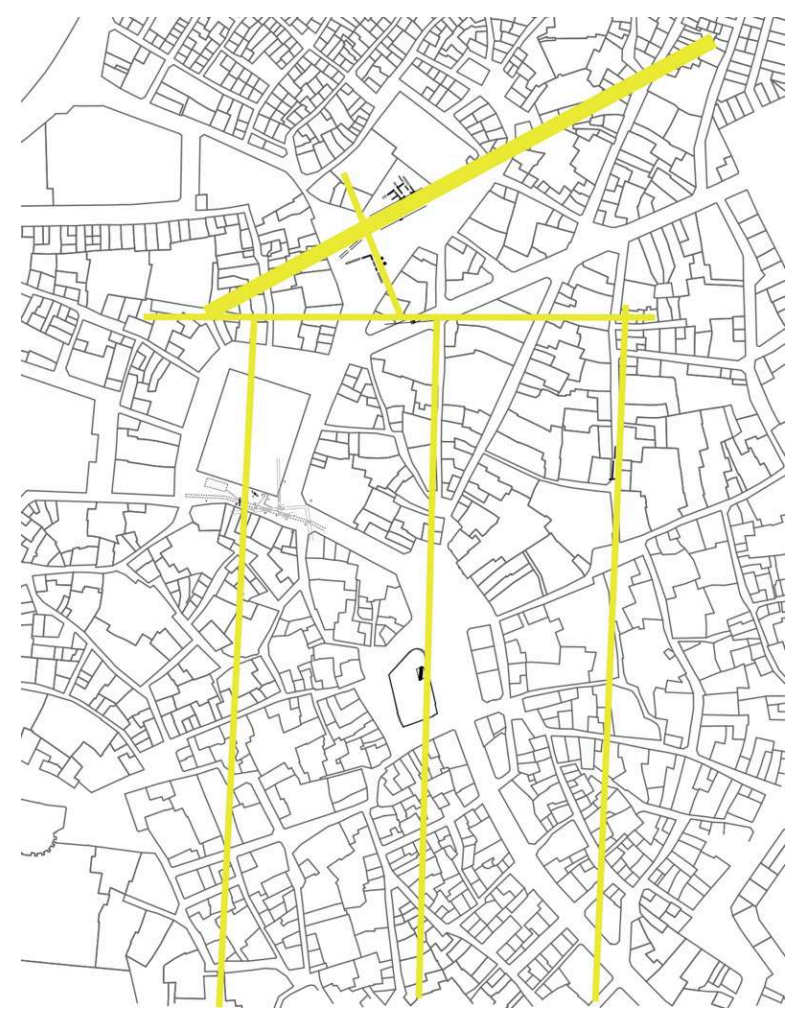

En jaune, axes de l'urbanisme grec.

Plan de C. Olive.

Pour l'heure, il n'y a pas de trace d'un rempart entourant la ville. Il aurait pu être utile du côté oriental, là où les fortifications médiévales et l'ampleur des travaux ultérieurs laissent peu de possibilités à la conservation d'une éventuelle courtine grecque.

Les édifices sont construits sur solins de pierre, parfois à orthostates, avec des élévations en adobes. Quelques cloisons internes sont simplement en adobes et le mur d'une boutique est en bauge. Les sols sont souvent en terre battue, parfois en adobes. Sont recensés aussi pavages et dallages en pierre, un sol étanche pour une cour interne, un sol à la chaux. Les foyers sont circulaires ou rectangulaires sur radier de tessons. Les rues présentent systématiquement un revêtement très dur composé de galets, pierres, scories de fer, tessons fortement compactés. L'emploi des tuiles en terre cuite est une originalité locale, car Béziers I/Rhòde a été la première et longtemps la seule ville du Nord-Ouest méditerranéen à les produire et à les utiliser largement pour la couverture des grands bâtiments et sans doute aussi, au moins en partie, pour l'habitat civil puisque les fragments se retrouvent en nombre partout. Plusieurs modules sont attestés, mais aucune pièce n'est entière ou graphiquement restituable (fig. 9). Ces tuiles informent aussi sur les toits: pour supporter des pièces qui devaient peser au minimum 7-8 kg chacune, ils étaient forcément charpentés de manière adaptée. 
Fig. 9. - Tuiles de courant et couvre-joints de production locale (fin $\mathrm{Vl}^{\mathrm{e}}-\mathrm{IV} \mathrm{V}^{\mathrm{e}} \mathrm{s}$.).
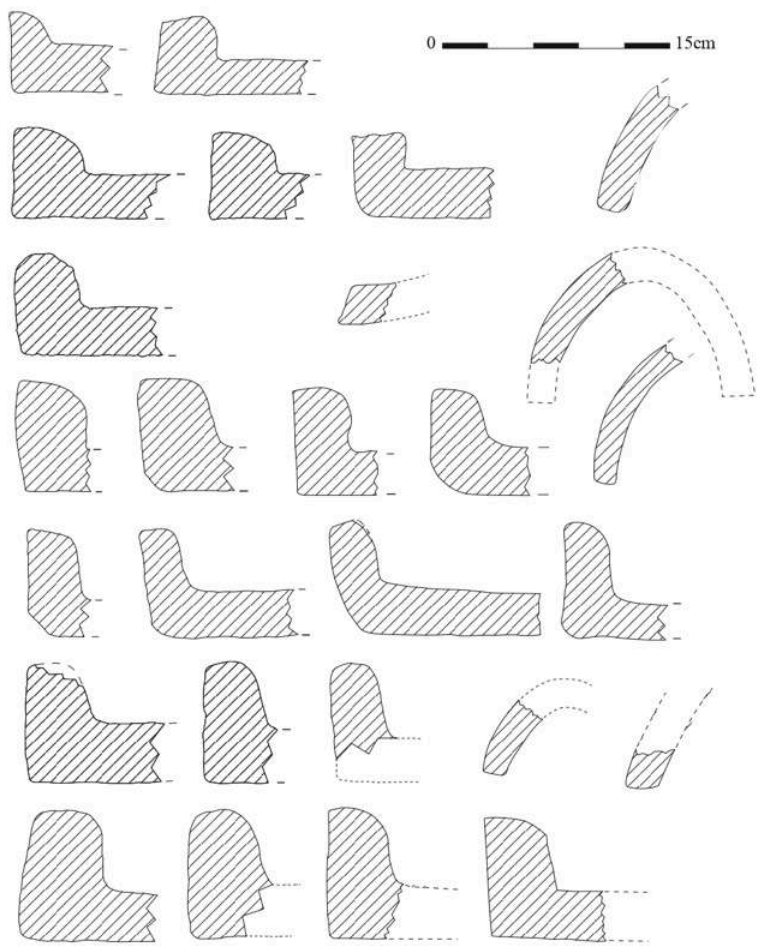

DAO de I. Fiches, M. Fresne, É. Gomez et C. Olive.

- Pour le vi $\mathrm{v}^{\mathrm{e}}$ siècle, sous les Halles (fig. $5, \mathrm{n}^{\text {os }} 3$ et 32 ), deux édifices à plan rectangulaire dont seul un angle pour chacun a été mis au jour semblent être des maisons, l'une de la deuxième moitié du siècle (au sud) et l'autre de la fin (au nord : fig. 10). Mal conservées et incomplètes, il est impossible de certifier un plan de type grec colonial, comme le fait qu'elles faisaient partie d'un îlot en regroupant plusieurs. L'une des deux (au sud) était bordée d'espaces ouverts, peut-être le croisement de deux rues ou venelles. 
Fig. 10. - Place Pierre Sémard 2017, sondage au nord des Halles. Angle d'une maison (?) de la fin du $\mathrm{VI}^{\mathrm{e}}$ siècle.

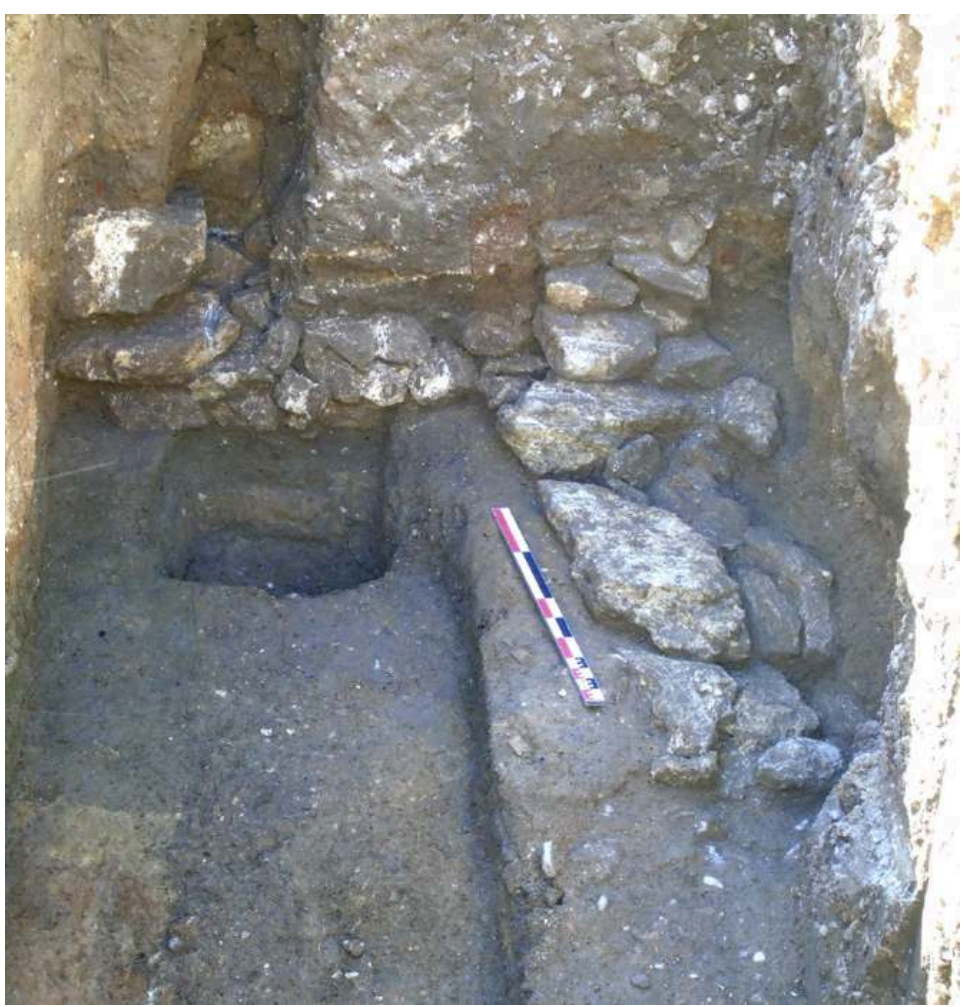

Cliché de É. Gomez.

27 - La maison de la place de la Madeleine (fig. $5, \mathrm{n}^{\circ} 1$ ), en bordure d'une très large rue $(9,60 \mathrm{~m})$, était également incomplète mais un peu moins que les deux autres. Trois limites sur quatre manquaient et la quatrième était incomplète. Elle occupait plus de $150 \mathrm{~m}^{2}$ au sol, avait plusieurs pièces, une cour interne au sol étanche et comportant vraisemblablement un puits, peut-être un étage. Elle a été en usage entre 460 et la fin $\mathrm{du} \mathrm{IV}^{\mathrm{e}}$ siècle et faisait partie d'un nouveau quartier et d'un îlot avec d'autres habitations ${ }^{16}$. Elle répond à une organisation domestique complexe et est encore - plus de trente ans après sa découverte - unique en Méditerranée nord-occidentale pour son époque ${ }^{17}$. Bien qu'incomplète, elle nous semble comparable aux maisons " à pastas ".

28 - D'autres vestiges sont attestés partout où des sondages ont été réalisés : tronçons de murs, de sols, de rues, foyers, fours domestiques...

29 L'artisanat est attesté très tôt et durablement. Les plus fréquents et les mieux documentés sont les ateliers des potiers, dont des vestiges sont présents en trois points de la ville et à la campagne pour un quatrième (voir ci-dessous). L'activité a été continue et la production (exclusivement tournée en ce qui concerne la vaisselle de table et de cuisine) intense pendant trois siècles.

30 - Îlot des Chaudronniers (fig. 5, $n^{\circ} 36$ ), un atelier de potier a fonctionné au tout début $\mathrm{du} \mathrm{VI}^{\mathrm{e}}$ siècle en bordure du lit originel de l'Embroucadou, généré par la puissante et toujours active source du Puits de L'Inquant qui sourd $120 \mathrm{~m}$ plus au nord (sous la place des Trois-Six aujourd'hui englobée dans la place du Forum). Les vestiges étaient conservés en-dessous des sols contemporains et seules les structures en creux ont pu être observées ${ }^{18}$ (fig. 11). 
Fig. 11. - îlot des Chaudronniers 2014. Plan des vestiges datés autour de 600 (d'après Macario, 2017, pl. 14bis).

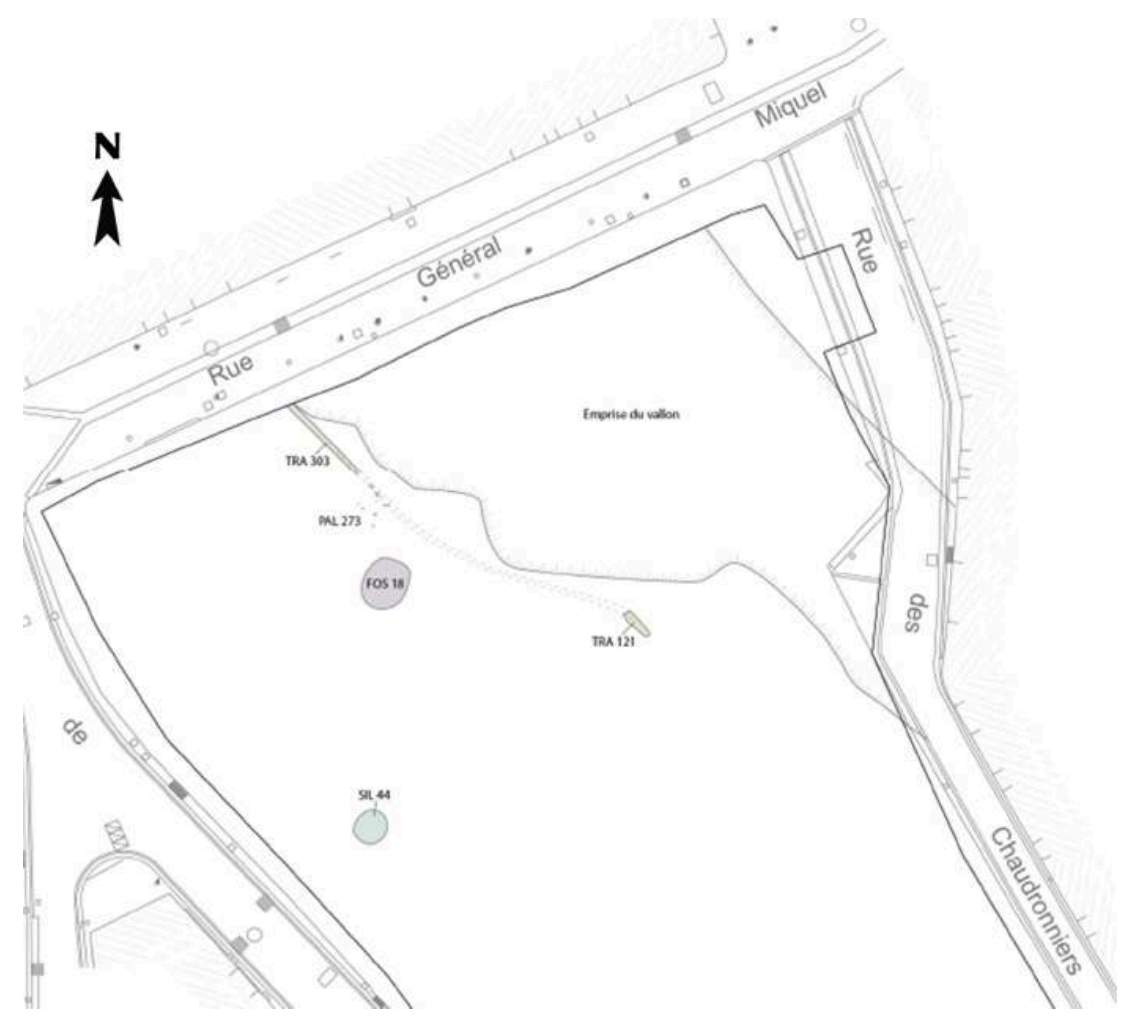

- En bordure du vallon, le fond d'une petite tranchée (TR303-121) marqué par de petits trous de piquets matérialisait une palissade légère.

- Le creusement 44 avait un fond plat et un profil tronconique ( $\varnothing$ ouverture : 0,85 $\mathrm{m} ; \varnothing$ fond : $2 \mathrm{~m}$; profondeur : $1,42 \mathrm{~m}$; volume : $\pm 3 \mathrm{~m}^{3}$ ). Amputé d'une partie de sa hauteur, il devait se terminer par un conduit cylindrique jusqu'à l'embouchure. Interprété comme un silo par le fouilleur (= SIL44), il s'agit en fait d'une citerne similaire à celle de la colline Saint-Jacques, mais de plus petite capacité, dont l'argile de l'encaissant assurait naturellement l'imperméabilité. Elle contenait la réserve d'eau du potier, puisée dans le vallon et stockée à proximité du lieu où il l'utilisait. Son comblement d'abandon comprenait les restes d'un four de potier démoli et arraché de son support originel, des vases non tournés, grecs importés et locaux ${ }^{19}$.

- Les fragments du four ont une face lissée et les traces de l'arrachement au revers (ép. max. conservée : 0,09 à 0,12 m) (fig. 12). La citerne ayant été fouillée pour moitié à la pelle mécanique, les fragments récupérés ne permettent pas de reconstituer entièrement le four, mais ses différentes parties peuvent être identifiées : ouverture voûtée de l'alandier (largeur ouverture: env. 0,50 m; hauteur totale estimée: autour de $0,65 \mathrm{~cm}$; épaisseur des fragments : 0,05 à 0,064 m) ; un fragment courbe du conduit excavé et arraché de l'alandier ; nombreux fragments de la paroi de la chambre de chauffe et de son fond circulaire $(\varnothing$ : autour de $0,90 \mathrm{~m})$; une pièce finie sur ses deux faces avec un large trou circulaire $(\varnothing: 0,16 \mathrm{~m})$ est l'évent d'une coupole qui devait être fixe et comporter une porte de charge ; un fragment de la sole perforée préserve un carneau ( des parois indiquent une certaine durée d'utilisation. Il est comparable à ceux des tablettes de Penteskouphia plus ou moins contemporaines ou, par exemple, à celui d'Olympie ( $\mathrm{v}^{\mathrm{e}}-\mathrm{IV}^{\mathrm{e}}$ siècle), et ses mesures réduites sont plutôt courantes durant la période archaïque ${ }^{20 .}$ 
Fig. 12. - îlot des Chaudronniers 2014. Embouchure de l'alandier, évent, fragment de la sole perforée, fragment du fond de la chambre de chauffe du four de potier daté vers 600 et proposition de restitution.

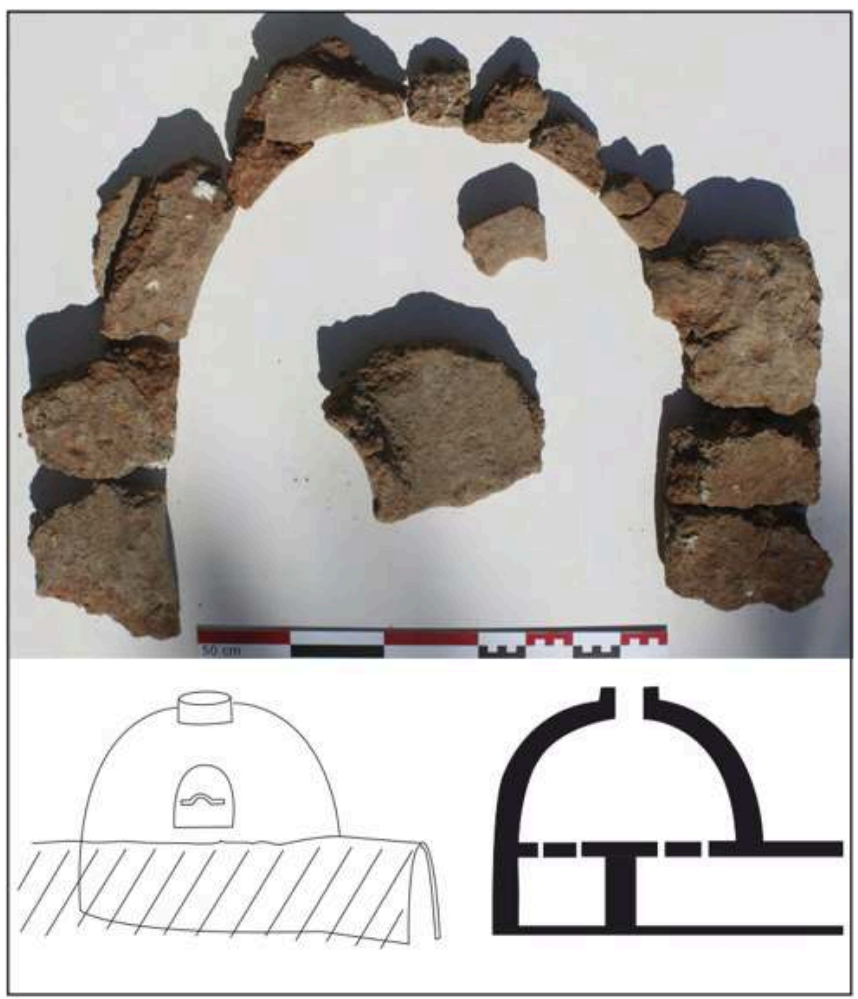

Cliché de É. Gomez ; DAO de D. Ugolini.

- La fosse 18, bouleversée par des creusements postérieurs et distante de $9 \mathrm{~m}$ de la citerne, avait une forme ovale. Ses mesures et ses parois, marquées par un épais liseré rubéfié, laissent supposer qu'il s'agit de l'emplacement primaire du four de potier, volontairement extirpé de son support originel.

- La paroi du vallon a été retaillée : sans doute par les potiers, qui attaquaient le banc d'argile à la pioche dans des situations analogues ${ }^{21}$.

Ces vestiges, aux marges méridionales de l'aire alors dévolue à l'habitat, illustrent le plus ancien atelier d'un potier grec en Méditerranée nord-occidentale ${ }^{22}$, implanté sur un banc d'argile parfaitement adaptée à la fabrication des vases et en bordure d'un cours d'eau. Ne manquaient que le combustible et les dégraissants qu'il était facile de se procurer alentour. La palissade de clôture suppose une organisation des accès et de la circulation : elle délimitait une aire qui devait être assez vaste, car l'activité exige de la place pour travailler l'argile, stocker les matières premières, tourner les vases, les laisser sécher, les cuire et les vendre.

- Sur la colline Saint-Jacques (fig. $5, n^{\circ} 33$ ), dans les comblements de la citerne et des premiers niveaux du grand fossé, ont été recueillis de nombreux fragments de fours de potier (parois circulaires avec d'épais revêtements rubéfiés, fragments de soles à carneaux, rebuts de cuisson). Ils attestent une activité se déroulant lorsque la fortification était encore en fonction, au début $\mathrm{du} \mathrm{VI}^{\mathrm{e}}$ siècle.

33 - Toujours sur la colline Saint-Jacques, après l'abandon de la fortification, le grand fossé a été progressivement comblé et dans ces sédiments des foyers en fosse se 
rapportant vraisemblablement à des forges de la deuxième moitié $\mathrm{VI}^{\mathrm{e}}$ siècle ont été observés.

$34-\mathrm{Au}$ nord de l'espace habité jusqu'à la fin du $\mathrm{VI}^{\mathrm{e}}$ siècle, place de la Madeleine (fig. 5, $\mathrm{n}^{\circ} 1$ ), en bordure de la source du ruisseau du Grazilhan (qui s'écoulait par le vallon de l'actuelle Porte Olivier), un atelier de potier, implanté sur un gisement de marnes adaptées à la fabrication des vases, comportait au moins deux fours, des fosses à dégraissants et une aire de vente ou de séchage. En activité durant le premier quart du $v^{e}$ siècle, il était longé par une sorte de piste préservant les ornières du passage d'engins à roues ${ }^{23}$.

35 - Dans la continuité de cet ensemble vers le nord, des multiples « foyers » ont été mis au jour dans des sondages anciens (fig. $5, \mathrm{n}^{\circ} 42$ ). Ils laissent supposer un artisanat d'une nature non précisée.

36 - Rue Mairan (fig. 5, $\mathrm{n}^{\circ}$ 21), un bas-fourneau du v viècle a été fouillé24. Il confirme, comme les forges de Saint-Jacques, une intense activité métallurgique qui se traduit aussi par les très nombreuses scories et loupes de fer relevées partout dans la composition des remblais. Et il y a évidemment les objets : clous de charpente, de porte, couteaux, outils, etc.

37 - À nouveau dans l'îlot des Chaudronniers (fig. $5, \mathrm{n}^{\circ}$ 36), dans le vallon déjà partiellement comblé de l'Embroucadou, dont les eaux avaient été détournées, des ateliers ont trouvé place à la fin du $\mathrm{v}^{\mathrm{e}}$ siècle :

- un bronzier utilisait le four 301 , excavé, à chambre ovalaire $(0,94 \times 0,80 \mathrm{~m})$ conservée sur $0,50 \mathrm{~m}$ de hauteur, avec un petit dôme percé d'une cheminée circulaire $(\varnothing: 0,30 \mathrm{~m})$, au pourtour rubéfié (0,05 à $0,10 \mathrm{~m}$ d'épaisseur) (fig. 13). Devant le four, l'aire de travail formait un goulot à l'entrée du four. Fragments de bronze, résidus cuivreux, charbons et cendres étaient associés aux vestiges abandonnés ${ }^{25}$. 
Fig. 13. - Îlot des Chaudronniers 2014. Plan de l'aire artisanale du dernier quart du ve siècle (d'après Macario, 2017, pl. 18).
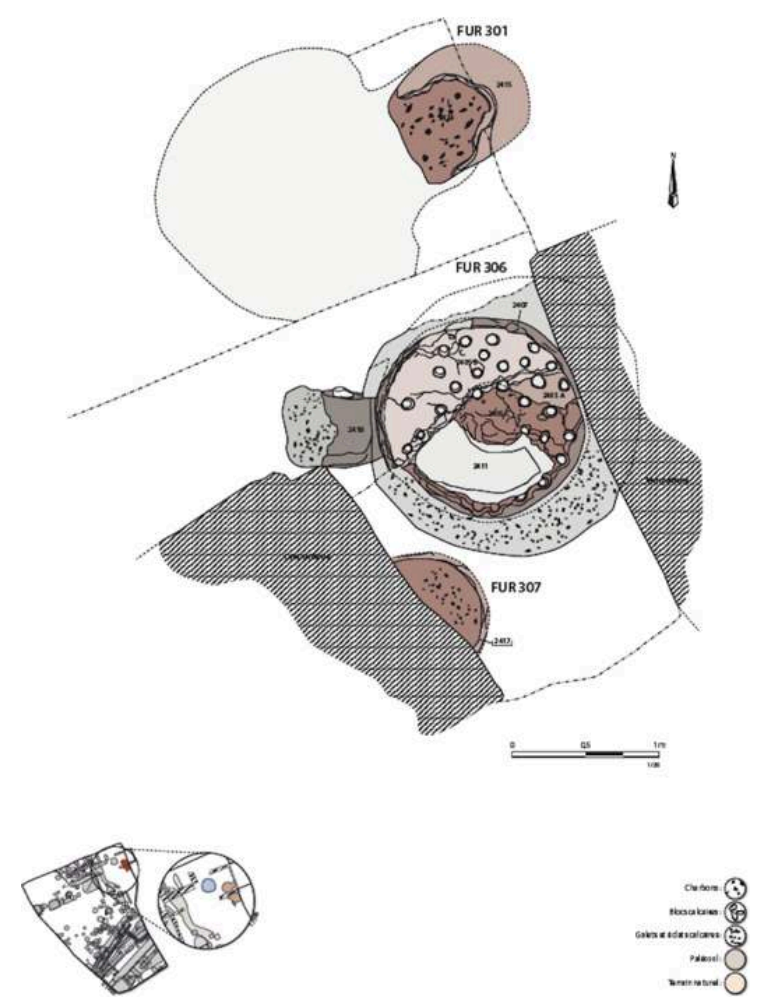

- Un potier utilisait le four 306, à chambre de chauffe excavée et pilier central en adobes supportant une sole partiellement en place $(\varnothing: 1,30 \mathrm{~m}$; profondeur originelle : 0,50-0,60 m et, après son approfondissement lors d'une réfection : $0,83 \mathrm{~m}$ ). Le départ de la coupole fixe $\mathrm{du}$ laboratoire était conservé sur une faible élévation. L'alandier était bâti, couvert par des adobes et obturé par une pierre (fig. 13). La fosse de travail s'ouvrait devant l'alandier. Trois phases de réfection témoignent d'une durée d'utilisation relativement longue avant l'abandon ${ }^{26}$. Le contexte situe l'activité vers 425-400.

- Du four 307, presque entièrement détruit par une cave moderne, il ne restait qu'une partie de la fosse ovale ( $\varnothing$ estimé : $\pm 0,90 \mathrm{~m}$; profondeur conservée : $0,40 \mathrm{~m}$ ), à la paroi enduite d'un revêtement et rubéfiée sur une épaisseur de $0,04 \mathrm{~m}$. Sa fonction est indéterminée ${ }^{27}$.

- Décalée vers le nord, la structure 308/US 2395 était une grande et épaisse plaque ovale de terre rubéfiée (1,80 x 1,50 m) correspondant au fond d'un grand four (ou foyer) arasé dont la fonction n'a pas été identifiée ${ }^{28}$.

Ces fours en plein centre-ville surprennent car leur fonctionnement pouvait causer des incendies. C'est pourquoi ces activités étaient situées de préférence à la périphérie des villes, comme c'est d'ailleurs le cas à Béziers même (aux marges de l'habitat du vi siècle pour l'atelier daté vers 600 de cet îlot et pour celui de la place de la Madeleine du début $\mathrm{du} \mathrm{v}^{\mathrm{e}}$ siècle). Toutefois, les officines n'étaient pas rares dans les villes grecques et souvent installées à proximité de lieux de culte ou de bâtiments publics en construction, pour les besoins de la finition architecturale des édifices et/ou pour la production et la vente de vaisselle, terres cuites figurées (ex-voto) ou autres objets nécessaires à la vie de ces espaces ${ }^{29}$. Elles pouvaient être éphémères ou, ayant trouvé un marché, perdurer. Nos fours se trouvent près du bâtiment de la fontaine alors en construction et ce n'est probablement pas par hasard si des fragments d'antéfixes et d'une plaque de revêtement architectural ont été retrouvés dans les comblements du 
vallon. Le fait que les fours sont grosso modo contemporains et ont été abandonnés plus ou moins en même temps plaiderait en faveur d'ateliers liés à la construction. Quant au risque d'incendie, leur position - en contre-bas des sols en fonction à la même époque à l'extérieur du vallon - ne présentait aucun danger pour le bâti environnant et permettait aux artisans un accès facile à l'argile et à l'eau qui, malgré la déviation du ruisseau, était proche.

- Une boutique, accolée au début du IV siècle à la maison de la place de la Madeleine (fig. $5, \mathrm{n}^{\circ} 1$ ), a rétréci la grande rue à une largeur de $5 \mathrm{~m}$. Un marchand échangeait ici du corail contre du bronze. Le commerce du corail est très présent dans la ville par la dispersion de branchettes brutes et/ou sciées et, en retour, par des objets continentaux en bronze. Les branchettes trouvées récemment dans des contextes datés autour de 600 font reculer d'autant les débuts d'une activité florissante qui, dans un premier temps et d'après la documentation alors disponible, avait été limitée aux $\mathrm{V}^{\mathrm{e}}$ et $\mathrm{IV}^{\mathrm{e}}$ siècles. Béziers $\mathrm{I} /$ Rhòde a donc été pendant très longtemps un intermédiaire principal entre Méditerranée et intérieur continental pour le trafic de cette matière, certainement dans le cadre de l'approvisionnement en bronze ${ }^{30}$.

Par la suite, cette boutique est devenue une boulangerie ${ }^{31}$.

D'autres artisanats sont attestés par des objets et par exemple celui sur les matières dures animales : os et cornes sciés, manches de couteaux, fléaux de balance de précision en os, etc.

La diversité et la continuité des activités artisanales sont désormais une évidence remarquable. Une telle concentration est unique et notamment celle concernant les potiers. Rappelons en effet que Béziers I/Rhòde est le seul site où des fours à vaisselle tournée (de table et de cuisine) ont été mis au jour ${ }^{32}$, ce qui renforce toujours davantage son rôle majeur dans la production et distribution de vases qui, trouvés dans d'autres sites, sont - hélas - régulièrement confondus avec d'autres ou dispersés sous des appellations incongrues, alors que le travail de ces potiers a permis la formation d'un faciès céramique bien caractérisé et différent de celui de Marseille ${ }^{33}$.

43 Les bâtiments publics sont - pour ainsi dire - les grands absents. Pas de chapiteaux, pas de colonnes, pas de statues, pas d'inscriptions..., mais quelques indices en laissent quand même supposer la présence.

44 - Les traces les plus sûres sont celles d'un édifice sans doute monumental aménageant l'écoulement de l'Embroucadou, réalisé autour de 425-400 (fig. 5, nº 30). Le tronçon d'un grand mur (épaisseur: $1,10 \mathrm{~m}$ ), bâti sur remblais pour le surélever par rapport à l'écoulement de la source et longé par une rue, a été repéré dans un sondage et de grands travaux ont déplacé le ruisseau (refluant d'abord dans le vallon naturel de l'îlot des Chaudronniers puis dévié vers la rue des Anciens Combattants) ${ }^{34}$. Cet édifice au cœur de la ville facilitait l'accès à une eau abondante d'excellente qualité, à la façon - pensons-nous - des fontaines grecques. Malheureusement, nous n'avons pas le plan de l'édifice, ni n'avons pu accéder au bassin, ni aux éventuels résidus de vidange : le système de distribution de l'eau est donc inconnu. Bien que partiels, ces vestiges témoignent d'un aménagement rare dans le $\mathrm{Midi}^{35}$.

$45-\mathrm{Au}$ sud des Halles (fig. 5, $\mathrm{n}^{\circ}$ 3), un curieux ensemble, en fonction entre les années autour de 500 et la fin du IV siècle, a été fouillé en $1986^{36}$. Il est caractérisé par des foyers et des fours qui se côtoient et se succèdent dans des espaces délimités par des cloisons (fig. 14). De grandes quantités de vaisselle de table, à feu et de préparation, 
d'amphores et de restes de faune accompagnaient ces vestiges que nous interprétons comme une "cuisine collective». Elle a pu servir les repas à l'occasion de banquets publics, civiques ou religieux, ou ceux d'une taverne/auberge, et semble sans comparaison dans le Midi.

Fig. 14. - Place Pierre Sémard 1986, sondage au sud des Halles. Foyers côte à côte, ve siècle.

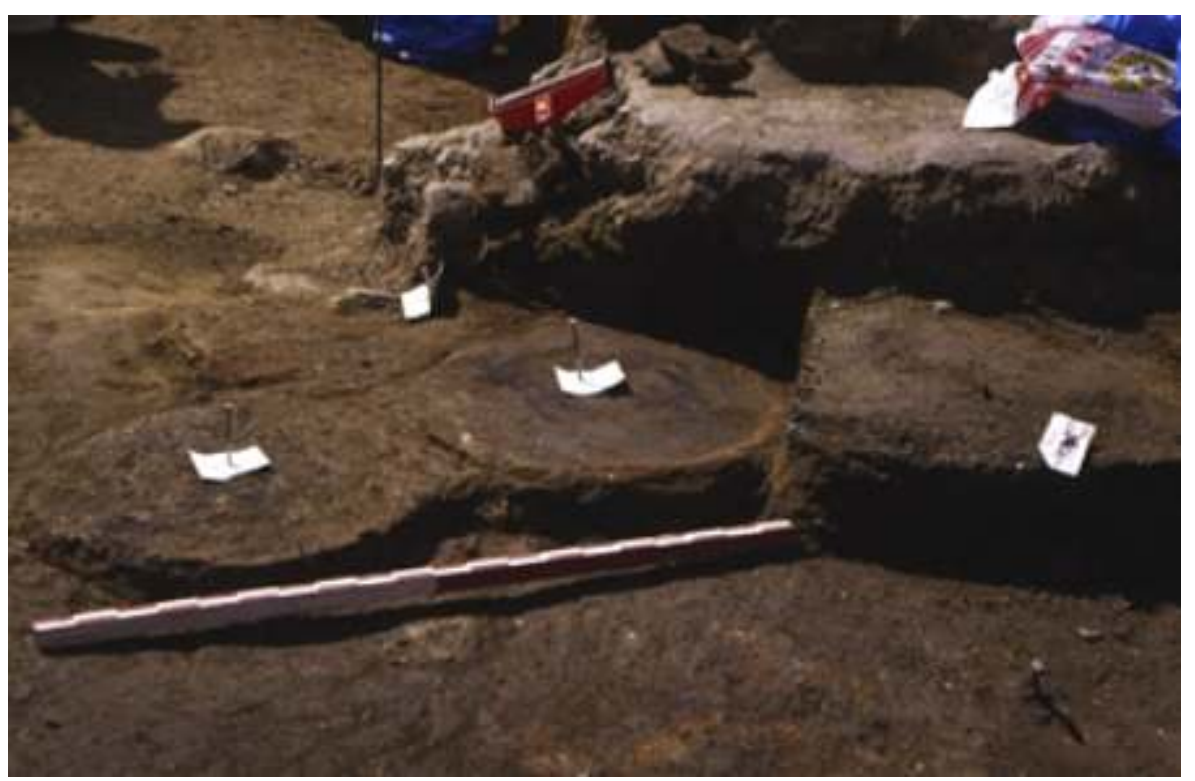

Cliché de J.-P. Wiégant.

- Tout à côté, a été trouvé hors contexte un fragment de poupée articulée. En terre cuite locale et de modèle " corinthien ", fréquent à partir de la fin $\mathrm{du}_{\mathrm{VI}}^{\mathrm{e}}$ siècle, notre pièce est la seule du Midi (fig. 15). Ces poupées, longtemps interprétées comme des jouets d'enfant, ne proviennent pas de contextes domestiques et, en revanche, caractérisent les dépôts votifs de divinités féminines en rapport avec la fécondité, la piété développée autour des sources et leurs figures tutélaires liées à la fécondité, les tombes de fillettes. D'ailleurs, les exemplaires les plus proches de Béziers sont ceux de deux tombes d'Emporion ${ }^{37}$. On considère aujourd'hui que ces poupées symbolisaient le passage de la jeune fille à la femme, lors de cérémonies au cours desquelles elles étaient offertes au temple/à la source, ou alors ce passage non advenu lorsque la fille décédait trop jeune. La présence de la poupée biterroise à proximité immédiate du jaillissement de la source du Puits de L'Inquant n'est sans doute pas fortuite, pourrait suggérer un lieu de culte proche, par exemple celui de la source, et représenter - ici comme ailleurs - une offrande votive. 
Fig. 15. - Place Pierre Sémard 1986, suivi de travaux au sud des Halles. Fragment de poupée articulée en terre cuite locale, deuxième moitié/fin $\mathrm{vl}^{\mathrm{e}}$ siècle - première moitié ve siècle.

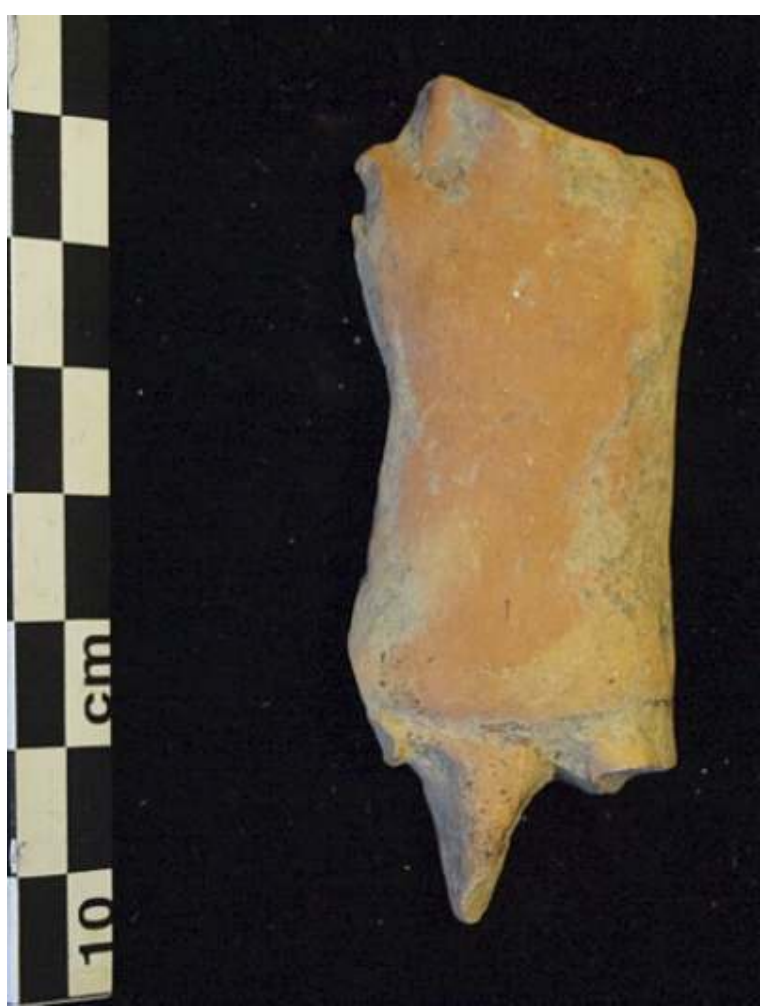

Cliché de É. Gomez.

47 - Une ou deux antéfixes et une plaque de revêtement architectural en terre cuite (fig. 16) décoraient forcément des monuments publics. 
Fig. 16. - Îlot des Chaudronniers 2014. Fragment de plaque de revêtement architectural en terre cuite locale, $v^{e}-I^{e} s .$, et essai de restitution.

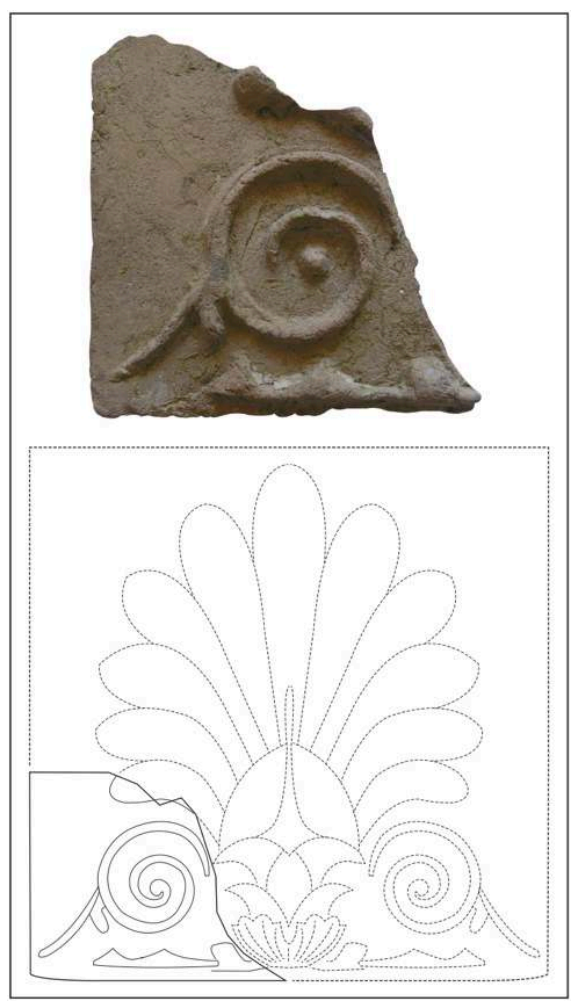

Cliché de É. Gomez ; DAO de D. Ugolini.

- Les tuiles en terre cuite de grand module étaient destinées à la couverture des grands édifices (fig. 9).

En attendant la découverte des lieux de culte, des pratiques religieuses de marque grecque se perçoivent par des objets caractéristiques :

- la poupée articulée ci-dessus ;

- un fragment de pinax en terre cuite massaliète préservant le bas du torse d'un personnage masculin (?) est vraisemblablement aussi un objet votif ;

- plusieurs vases miniatures en céramique claire et grise monochrome biterroises ont été trouvés dispersés dans la ville. Objets courants dans les nécropoles et les sanctuaires, ils étaient utilisés aussi pour des dépôts de fondation dont quelques exemples sont connus dans le Midi $^{38}$;

- quelques phialai attiques et loutéria massaliètes à décor en relief ont pu servir dans certains rituels ;

- des objets en terre-cuite locale que nous interprétons comme des autels portatifs sont originaux et uniques dans le Nord-Ouest méditerranéen. Très fragmentés et très épais, leur forme ne peut être restituée : il s'agit de pièces de petite taille obtenues à partir de pains d'argile modelés ou moulés. Ils portent des décors en léger relief imprimés avec des matrices à cylindre et des tampons et peut-être aussi moulés. Trouvés dispersés dans les remblais de toutes les zones de la ville, leur utilisation devait être courante, voire systématique entre le $\mathrm{VI}^{\mathrm{e}}$ et le $\mathrm{IV}^{\mathrm{e}}$ siècles. Si l'identification fonctionnelle que nous proposons est la bonne, nos autels sont domestiques et typiques d'une pratique très développée, avant l'époque hellénistique, surtout en Grande-Grèce et Sicile. Du point de vue décoratif, les nôtres sont 
modestes (frises de cavaliers, méandres, grecques, palmettes, cercles concentriques, roues, animaux) mais rappellent les exemplaires italiens ${ }^{39}$ (fig. 17).

Fig. 17. - Fragments d'autels domestiques en terre cuite locale avec décors imprimés, trouvés en plusieurs endroits de la ville, $\mathrm{Vl}^{\mathrm{e}}-\mathrm{IV} \mathrm{e}^{\mathrm{e}} \mathrm{s}$.

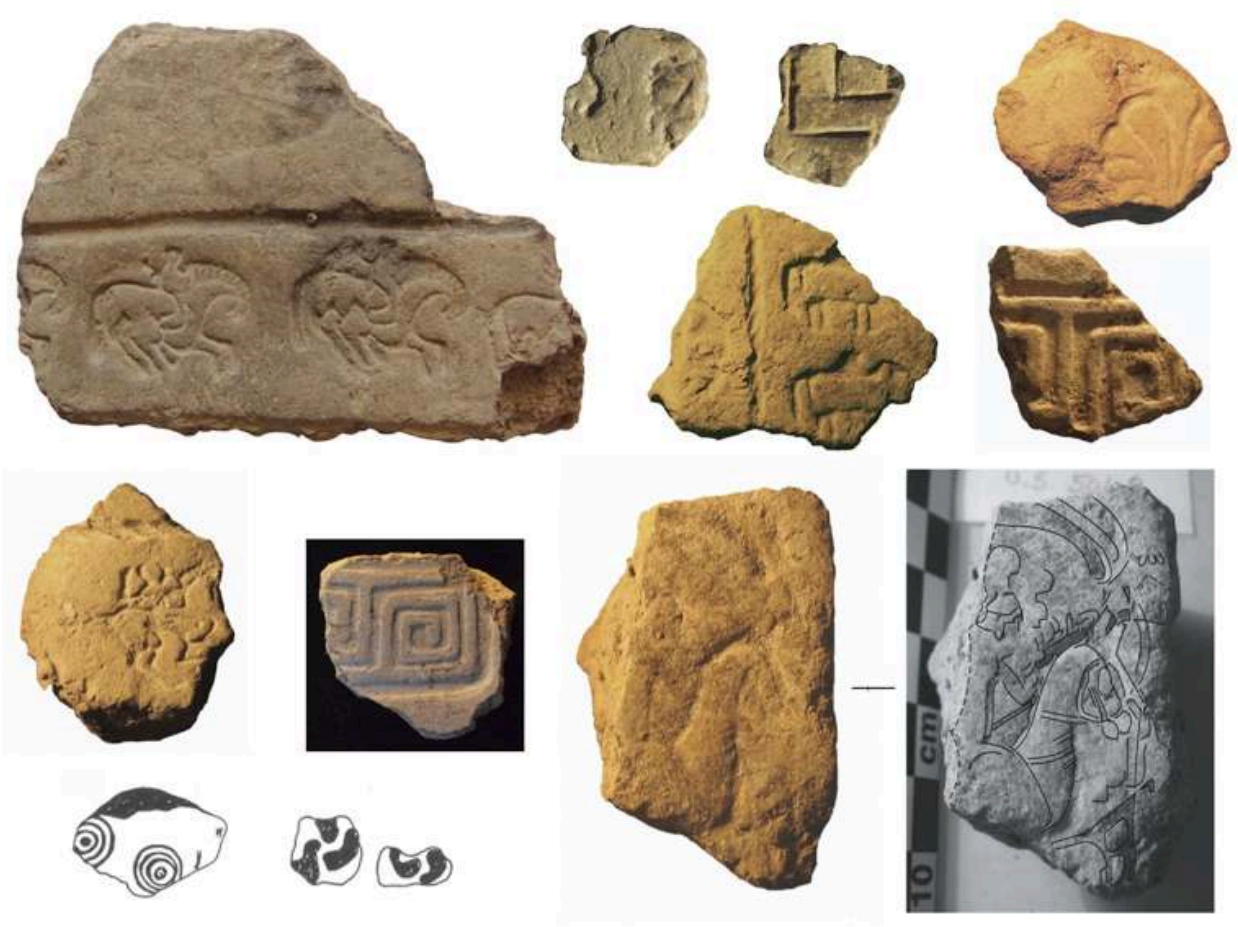

Clichés de M. Fresne, É. Gomez et C. Olive ; dessins de C. Lapeyre.

\section{La chôra}

Autre aspect fondamental de la ville grecque, la chôra est perceptible dès le milieu du $\mathrm{VI}^{\mathrm{e}}$ siècle ${ }^{40}$ (fig. 18). La délimiter avec certitude n'est pas simple en l'absence des lieux de culte « de frontière » qui nous y aideraient. 
Fig. 18. - La chôra de Béziers I/Rhòde.

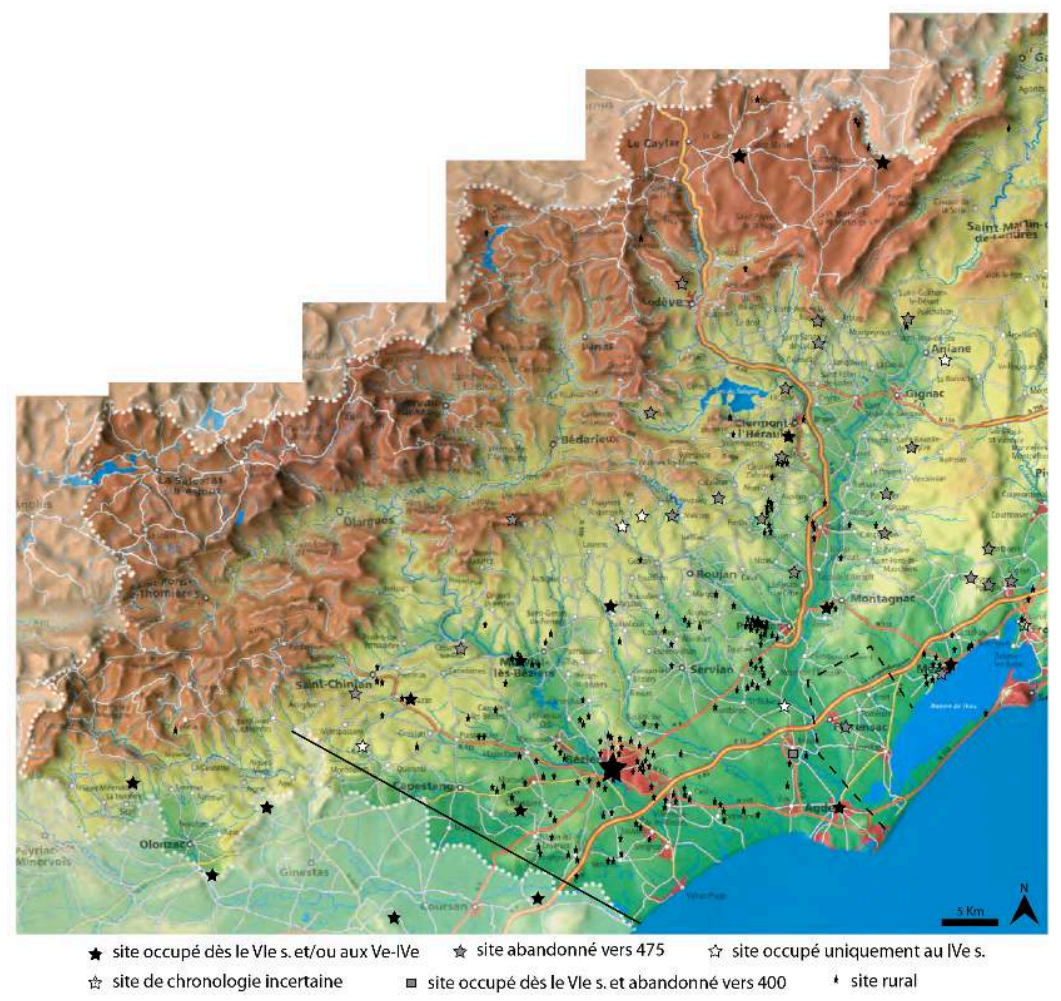

Les nombreux sites ruraux datés entre 525 et 475 en liaison avec Mont-Joui (aire en pointillé) ne sont pas reportés sur la carte (voir Gomez, 2010).

Données sur carte Géorelief34 par É. Gomez, C. Olive et D. Ugolini.

Le terroir vivrier se perçoit par les soixante-six sites ruraux identifiés à ce jour autour de la ville, sur les deux rives du fleuve. En posant théoriquement la limite orientale au fleuve Libron, l'occidentale à mi-chemin entre Béziers et Ensérune et la septentrionale quelque part entre la ville et les agglomérations proches, Béziers I/Rhòde gérait et exploitait assurément en propre un espace d'environ $260-280 \mathrm{~km}^{2}$. Cette estimation ne tient pas compte de sites ruraux plus éloignés ne se trouvant pas dans l'environnement d'une autre agglomération, qui ont dû faire partie de ce territoire, au moins jusqu'aux communes de Valros, Servian, Corneilhan, Puisserguier, Vendres, Vias, Agde. Ils amènent la superficie à plus de $400 \mathrm{~km}^{2}$. De sa grande taille et de la densité déjà élevée des sites repérés - qui s'accroît depuis que l'on y prête attention-, on déduit l'importance de l'investissement et, par conséquent, le nombre considérable de personnes actives dans cette campagne. Ces sites ne sont pas tous contemporains entre eux, mais appartiennent tous à la phase grecque de Béziers, notamment aux deux siècles de sa meilleure période (550-350), et sont marqués par la présence récurrente et plus ou moins importante des céramiques de la ville. La majorité d'entre eux n'est connue que par des prospections ne permettant pas d'en établir la nature, mais d'autres ont été fouillés en totalité ou en partie : fermes, épandages dans des champs cultivés, vignes (la plus ancienne - La Courondelle - en fonction à partir de 500, l'autre - Le Frigoulas - au $v^{e}$ siècle ${ }^{41}$ ), fosses, puits, fossés agraires, chemins, fréquentations de sources et grottes, un four de potier faisant sans doute partie d'une grande exploitation. 
Ce dernier, $3 \mathrm{~km}$ à l'est de la ville et en bordure de la vallée du ruisseau de Mazeran riche de marnes et sables adaptés à la fabrication des vases, longeait l'axe qui sera celui de la voie Domitienne (et qui était probablement déjà celui de l'Héracléenne). Grand (Ø interne : 2,65 m ; profondeur conservée : $0,84 \mathrm{~m}$ ), conservé sous la sole (circulaire percée de carneaux) soutenue par trois supports en adobes disposés en croix sur le fond de la chambre de chauffe et terminés, contre la paroi, par des voûtes en encorbellement, il a fonctionné durant les premières décennies du IV $\mathrm{I}^{\mathrm{e}}$ siècle $^{42}$. Le dispositif cruciforme et les fausses voûtes ont été observés également à Marseille pour le four à amphores de la rue Leca ( $v^{\mathrm{e}}$ siècle). Notre four était spécialisé dans la cuisson de pithoi à la pâte caractéristique (nombreux surcuits et sous-cuits). C'est le seul dévolu à cette fonction connu dans le monde grec, où les grands fours cuisaient indistinctement diverses pièces de grande taille. Notons toutefois que des surcuits d'amphores massaliètes proviennent du comblement. Nous avons préféré n'y voir qu'un accident, mais - avec toute la circonspection qui s'impose - n'écartons pas la possibilité que des amphores semblables aux massaliètes aient pu y être cuites, ce qui soulève une question épineuse nécessitant des arguments plus probants pour éviter toute polémique. Il reste que la ville a produit du vin, comme le prouvent à la fois les vignes déjà localisées et les pithoi poissés, présents partout et tout le temps, dont les plus anciens ont été trouvés dans des contextes datés autour de 600 (en deux endroits différents). Comme il est impensable que l'on ait stocké dans ces récipients du vin venu d'ailleurs, on en déduit que la viticulture a été précoce. Au début la production devait être limitée et réservée à la consommation locale, mais même après, lorsque les vignobles ont été étendus, la ville n'a pas mis au point un récipient de transport propre et différent. Par prudence, nous laisserons de côté les surcuits trouvés dans ce four pour avancer l'hypothèse - certes un peu surprenante - que le vin biterrois ait été conditionné dans des amphores massaliètes arrivées vides pour y être remplies et distribuées alentour. Elle nous parait envisageable tant ces récipients sont abondants en ville ${ }^{43}$ et en Biterrois. D'un autre côté, est-il possible que Marseille ait fourni seule tout le vin contenu dans les milliers d'amphores distribuées? D'autres sites ont dû contribuer à la production et certainement Béziers I/Rhòde, qui avait un grand territoire et des vignobles. Le fait que la seule amphore utilisée pour le transport du vin du Midi est la massaliète, ne laisse finalement que deux possibilités : soit les amphores «massaliètes » n'étaient pas fabriquées qu'à Marseille, soit le vin produit ailleurs qu'à Marseille était conditionné dans des amphores massaliètes vendues au moins en partie vides, en tant qu'emballages.

La diversité des traces rurales traduit la complémentarité des activités exercées dans ces campagnes. Le cas est remarquable car autour de Marseille, d'Agde et d'Emporion, où les territoires respectifs ont été étudiés, rien d'analogue n'est connu et l'appréciation de ces chôrai est basée sur des approches théoriques ${ }^{44}$. Malheureusement, le bâti est toujours en très mauvais état, voire a disparu à cause du faible recouvrement et de l'intensité des labours depuis l'Antiquité. Pour l'heure, il n'est donc pas possible d'identifier des fermes au plan typiquement grec, ni d'affirmer que seuls des Grecs exploitaient ces terres, mais les vestiges sont le plus souvent sous les/à proximité immédiate des villas romaines dont l'installation a détruit ce qui restait des constructions grecques. Ce constat pourrait suggérer une organisation des terres que les cadastres d'époque romaine - très étudiés en Biterrois - ont peu ou prou calquée. 

compter sur un "domaine " plus vaste garantissant l'accumulation de surplus pour l'échange et la circulation des marchandises. Cette acception élargie de la chôra pose la question du rôle qu'il convient d'envisager pour les agglomérations périphériques, dont les plus proches sont disposées quasiment en cercle autour de la ville, à une distance moyenne d'une vingtaine de kilomètres et contrôlent chacune une voie de passage. Indépendamment de l'ethnie de leurs habitants, il est assuré que quelques-unes ont été créées au fil du temps pour seconder les dynamiques de Béziers I/Rhòde, pour structurer les contacts avec les indigènes, pour favoriser la circulation des hommes et des biens, pour maîtriser l'accès à la mer (Agde, La Monédière/Bessan, Mus/Murviellès-Béziers, Le Fort/Saint-Thibéry, peut-être Ensérune/Nissan-lez-Ensérune et Mèze). D'autres, non comprises dans le territoire dans un premier temps, ont fini par disparaître (p. ex. Mont-Joui/Florensac) et il est possible que la ville ait alors englobé leurs terres. Enfin, pour certaines plus éloignées l'incertitude demeure.

Lappréciation de la chôra dans ses divers aspects reste ainsi ardue là où Grecs et indigènes ont coexisté et trouvé un mode de fonctionnement qui, forcément, nous échappe en grande partie. S'il est évident que les lignes ont bougé au cours du temps au profit des Grecs, dans l'immédiat nous nous contenterons de relever qu'autour de 500 et en s'en tenant aux données les plus sûres, les vallées de l'Hérault, de la Peyne, du Libron, de l'Orb, du Lirou et de l'Aude étaient au cœur des activités économiques de la ville, qui ciblait - outre l'échange côtier - un commerce continental dont le préalable indispensable était un réseau de chemins sûr. C'est d'ailleurs lorsque ce réseau n'a plus fonctionné que se sont produits d'abord l'effondrement économique de la ville puis son abandon, autour de 300 .

\section{Conclusion}

De ce rapide survol se dégage que la première Rhòde d'Occident a été d'abord un petit établissement plus ou moins contemporain, ou dans la suite immédiate, des premières importations grecques du Midi (datées du troisième quart du vile siècle) mises au jour entre l'Hérault et l'Aude et à Béziers même. Le temps qui s'écoule entre l'exploration préalable et l'installation grecque semble donc avoir été court. Ouverte aux indigènes qui ont permis, par leurs réseaux, d'instaurer la collecte du bronze - matérialisée par les dépôts launaciens et par les objets parvenus en Sicile et ailleurs -, au début, elle a pu être un simple emporion créé pour faciliter l'échange ${ }^{45}$. Toutefois, l'organisation de la ville, qui se peuple et s'agrandit, s'amorce rapidement et met en place une chôra. Elle devient donc, selon toute vraisemblance et assez vite, une colonie (apoikia). Les relations avec les indigènes sont réorganisées au fil du temps mais finissent par se dégrader, notamment après la création rhodo-biterroise du port d'Agde I/Agàthe, à la toute fin $\mathrm{du} \mathrm{vI}^{\mathrm{e}}$ siècle ${ }^{46}$.

D'autres sites occidentaux étaient peut-être aussi de souche principalement dorienne : la non localisée Rhodanousia et Arles/Theline (si ces deux sites ne correspondent pas à un seul) et aussi Collioure/Pyréne $e^{47}$. Quant à Roses/Rhòde, sa fondation intervient dans le contexte d'une crise de Béziers I/Rhòde qui va s'aggraver jusqu'aux extrêmes conséquences et qui justifie le déplacement d'une partie des habitants vers la nouvelle Rhòde. Tous ces sites ont contribué à construire un espace grec particulier à l'ouest du Rhône, parallèle à celui ionio-phocéen de l'est. La complémentarité au moins 
économique entre les deux domaines était indispensable et le fait est qu'on ne relève pas de conflits pendant trois siècles entre les deux villes principales ${ }^{48}$.

La colonie a atteint un bon niveau de prospérité. L'importance des trafics se saisit, d'une part, par la distribution de ses vases, qui joue un rôle de premier plan dans l'identification de son rayon d'action et d'influence; d'autre part, par la quantité, variété et qualité des importations; et, enfin, par des choix particuliers. Par exemple, au v $v^{\mathrm{e}}$ siècle, lorsqu'elle est rare ailleurs, la céramique attique est abondante (comme à La Monédière, site étroitement lié à Béziers) et décroît au IV siècle, lorsqu'elle est largement présente dans les sites indigènes. Les amphores étrusques, nombreuses dans les niveaux anciens, arrivent encore lorsqu'elles ont quasiment disparu ailleurs. Le bucchero nero, courant au $\mathrm{VI}^{\mathrm{e}}$ siècle, ne l'est pas ici et l'étrusco-corinthienne n'est pas encore attestée. En revanche, les mortiers étrusques étaient achetés pour leur diamètre "grand", non proposé par les ateliers locaux, et les massaliètes vraisemblablement pour leur prix inférieur à celui des biterrois. Ces choix originaux témoignent de l'ouverture commerciale de la place, qui n'était sous aucun monopole. Ainsi, importations et productions locales ont contribué à la formation et à la progression d'un mode de vie singulier qui a duré jusqu'à la récession amorcée vers 350 et finalement soldée par l'abandon autour de 300.

Bien que les bâtiments publics ne soient pas encore assurés et les nécropoles localisées, les informations disponibles ont un poids déjà considérable et orientent vers le développement d'une véritable pòlis, composée d'une asty et d'une chôra dont les vestiges respectifs émergent au fur et à mesure des recherches, selon un schéma classique qui n'a pourtant pas de réelle comparaison dans le Nord-Ouest méditerranéen de son temps. Elle a durablement marqué le Midi par son investissement régional, son impact le long de la côte, ses relations méditerranéennes à longue distance, ses contacts avec l'intérieur continental.

\section{BIBLIOGRAPHIE}

AQUILUÉ Xavier, CASTANYER Pere, SANTOS Marta \& TREMOLEDA Joaquim, « Les ceràmiques gregues arcaiques de la Palaià Polis d'Empòrion », dans P. Cabrera Bonet (éd.), Ceràmiques jònies d'època arcaica: centres de producció i comercialització al Mediterrani occidental (Actes de la table ronde d’Empúries, mai 1999), Barcelone, Generalitat de Catalunya, 2001, p. 285-346.

BOUFFIER Sophie \& GARCIA Dominique (éd.), Les territoires de Marseille antique, Paris, PUF, 2014.

CAG 34/4 = UGOLINI Daniela \& OLIVE Christian, avec la collaboration de GOMEZ Élian (éd.), Béziers. Carte archéologique de la Gaule, 34/4, Paris, Académie des Inscriptions et Belles-Lettres, 2012.

CALDERONE Anna, «Il mito greco e le arule siceliote di VI-V sec. a.C. », dans Le mythe grec dans l'Italie antique. Fonction et image (Actes du colloque international, Rome, 14-16 novembre 1996), Rome, École française de Rome, 1999, p. 163-204. 
DIETLER Michael, KOHN Alison, MOYA I GARRA Andreu \& RIVALAN André, « Les maisons à cour des III $^{\mathrm{e}}-\mathrm{II}^{\mathrm{e}} \mathrm{s}$. av. J.-C. à Lattes : émergence d'une différenciation dans l'habitat indigène ", Gallia, 65, 2008, p. 111-122.

DUNYACH Ingrid, La place du Roussillon dans les échanges en Méditerranée aux âges du Fer. Étude d'une organisation territoriale, sociale et culturelle ( $\mathrm{VI}^{e}-\mathrm{III}^{e}$ siècle avant $J .-\mathrm{C}$.), thèse de doctorat, Université de Perpignan-Via Domitia, 2018.

ESPOSITO Arianna, « La question des implantations grecques et des contacts précoloniaux en Italie du Sud : entre emporia et apoikiai », dans L. Martinez-Sève (éd.), Les diasporas grecques du VIII à la fin du III siècle av. J.-C., Pallas, 89, 2012, p. 97-121.

ESPOSITO Arianna \& SANIDAS Giorgios (éd.), Quartiers artisanaux en Grèce ancienne. Une perspective méditerranéenne, Villeneuve-d'Ascq, Presses universitaires du Septentrion, 2012.

FERNANDEZ-GÖTZ Manuel, « Le Danube et la Heuneburg : histoire d'une rivière », dans F. Olmer \& R. Roure (éd.), Les Gaulois au fil de l'eau (Actes du $37^{\mathrm{e}}$ colloque de l'AFEAF, Montpellier, 2013), vol. 1, Bordeaux, Ausonius, 2015, p. 259-271.

GAILLEDRAT Éric, ANWAR Nasrine, BEYLIER Alexandre, BRUNNER Philippe, CAMMAS Cécilia, CAVERO Julian, CURE Anne-Marie, DEVILLERS Benoît, DUDAY Henri, FAISSE Camille, GARDEISEN Armelle, MARCHAND Georges, MUNOS Sébastien, PIQUÈS Gaël, PROVENZANO Noëlle, ROUX JeanClaude, SANZ Séverine \& VACHERET Ariane, Pech Maho (Sigean, Aude). Rapport de fouille 2010. Disponible en ligne sur <https://halshs.archives-ouvertes.fr/halshs-00560425v2>.

GOMEZ Élian, Agde et son territoire, thèse de doctorat, Université d'Aix-Marseille, Aix-en-Provence, 2010.

GOMEZ Élian, UGOLINI Daniela \& CROS Jean-Paul, « Béziers : aux origines de la plus ancienne ville grecque de France », Archéologia, 568, 2018, p. 30-35.

GUILAINE Jean, CAROZZA Laurent, GARCIA Dominique, GASCÒ Jean, JANIN Thierry \& MILLE Bernard, Launac et le launacien, Montpellier, PULM, 2017.

HASAKI Eleni, Ceramic Kilns in Ancient Greece: Technology and Organization of Ceramic Workshops, thèse de doctorat, Université de Cincinnati, 2002.

KUNZE Emil, SCHLEIF Hans \& EILMANN Richard, Bericht über die Ausgrabungen in Olympia, 3, Winter 1938/1939, Berlin, De Gruyter, 1939.

LA ROCCA Luigi, «Su un gruppo di arule nel Museo Archeologico di Crotone », Prospettiva, 85, 1998, p. 39-45.

LA ROCCA Luigi « Arule e ceramiche a rilievo di produzione crotoniate », dans R. Belli Pasqua \& R. Spadea (éd.), Kroton e il suo territorio tra VI e V secolo a.C. (Atti del convegno di studi, Crotone, 3-5 marzo 2000), Crotone, Comune di Crotone, 2005, p. 43-54.

MACARIO Raphaël (éd.), îlot des Chaudronniers, Béziers, Hérault. HADES, Rapport final d'opération archéologique, 5 vol., Toulouse, HADES, 2017.

MARTINEZ-SÈVE Laurianne (éd.), Les diasporas grecques du VIII à la fin du III siècle av. J.-C., Pallas, 89, 2012.

MEIJDEN Hellebora van der, Terrakotta-Arulae aus Sizilien und Unteritalien, Amsterdam, Adolf M. Hakkert, 1993. 
OLIVE Christian \& UGOLINI Daniela, « La Maison 1 de Béziers et son environnement $\left(\mathrm{V}^{\mathrm{e}}-\mathrm{IV} \mathrm{e}^{\mathrm{e}} \mathrm{s}\right.$. av. J.-C.), dans D. Ugolini (éd.), Languedoc occidental protohistorique. Fouilles et recherches récentes ( $\mathrm{VI}^{e}$ IV s. av. J.-C.), Aix-en-Provence, PUP, 1997, p. 87-129.

OLIVE Christian, UGOLINI Daniela \& RATSIMBA Antoine, « Un four de potier pour la cuisson de pithoi à Béziers (Hérault). Production, diffusion et fonction du pithos dans le Midi ( $\mathrm{VI}^{\mathrm{e}}-\mathrm{IV}^{\mathrm{e}} \mathrm{s}$. av. J.-C.) », Gallia, 66, 2009, p. 29-57.

PALMIERI Maria Grazia, Siti di produzione ceramica nel mondo greco dalla prima Età del ferro all'Età ellenistica, thèse de doctorat, Università Federico II, Naples, 2013.

PAPAIKONOMOU Irini-Despina, «Enfance et identité sexuée dans les cités grecques », dans F. Gusi, S. Muriel \& C. Olària (éd.), Nasciturus, infans, puerulus vobis mater terra. La muerte en la infancia, Castellò, SIAP, 2008, p. 683-710.

PEZIN Annie, « Les fours de potier du Mas de Pascal à Aspiran (Hérault) », dans J.-P. Demoule (éd.), La France archéologique. 20 ans d'aménagements et de découvertes, Paris, Hazan, 2004, p. 115.

POLIGNAC François de, «L'installation des dieux et la genèse des cités en Grèce d'Occident, une question résolue ? Retour à Mégara Hyblaea ", dans La colonisation grecque en Méditerranée Occidentale, Rome, École française de Rome, 1999, p. 209-230.

PONSICH Pierre, « De l'homme de Tautavel au Roussillon moderne », Archéologia, 83, 1975, p. 10-15.

RONDI-COSTANZO Cecilia, « Corail de Béziers, du Midi de la Gaule et de Méditerranée », dans D. Ugolini (éd.), Languedoc occidental protohistorique. Fouilles et recherches récentes (VI $I^{e} I V^{e}$ s. av. J.-C.), Aix-en-Provence, PUP, 1997, p. 197-239.

RONDI-COSTANZO Cecilia \& UGOLINI Daniela, « Le corail dans le bassin nord-occidental de la Méditerranée entre le $\mathrm{VI}^{\mathrm{e}}$ et le $\mathrm{II}^{\mathrm{e}}$ s. av. J.-C. », dans J.-P. Morel, C. Rondi-Costanzo \& D. Ugolini (éd.), Corallo di ieri, corallo di oggi (Atti del convegno di Ravello, 13-15 dicembre 1996), Bari, Edipuglia, 2000, p. 177-191.

ROURE Réjane, JONCHERAY Claire, OLLIVIER David, ESCOFFON Pierre, DEAL Carine, SALLE Valérie \& SÉRIEYS Maeva, « Un dépôt de fondation en contexte domestique à Olbia de Provence (Hyères, Var)», dans R. Roure (éd.), Contacts et acculturations en Méditerranée occidentale. Hommages à Michel Bats (Actes du colloque de Hyères, 15-18 septembre 2011), Arles, Actes Sud, 2015, p. 99-109.

TRÉZINY Henri, «Fossés et défenses avancées dans les villes grecques d'Occident », Revista d'Arqueologì de Ponent, 21, 2011, p. 287-296.

TRÉZINY Henri, « Marseille antique. Topographie, urbanisme, architecture ", Empùries, 56, 2009-2011, p. 41-54.

UGOLINI Daniela (éd.), Languedoc occidental protohistorique. Fouilles et recherches récentes ( $V I^{e}-I V^{e} s . a v$. J.-C.), Aix-en-Provence, PUP, 1997.

UGOLINI Daniela, «Le Roussillon : passage obligé des trafics en Méditerranée nord-occidentale », dans Comerç i vies de communicaciò (Actes du XI ${ }^{\mathrm{e}}$ colloque international de Puigcerdà, 1997), Puigcerdà, Inst. d'Estudis Ceretans, 1998, p. 73-92.

UGOLINI Daniela, « Les Ibères des Pyrénées au Rhône. Bilan de 20 ans de recherches », dans Mon ibèric als Països Catalans. Homenatge a Josep Barberà i Farràs (Actes du XIII ${ }^{\mathrm{e}}$ colloque international de Puigcerdà, 2003), Puigcerdà, Inst. d'Estudis Ceretans, 2005, vol. I, p. 165-202.

UGOLINI Daniela, « Il corallo tra il VII e il II secolo a.C. lungo le coste della Catalogna e della Gallia », dans Coralli segreti. Immagini e miti dal mare tra Oriente e Occidente, Catalogue de 
l'exposition, Potenza, Museo Archeologico Nazionale della Basilicata 'Dinu Adamesteanu', 2006, p. 78-87.

UGOLINI Daniela, « Béziers I : entre Méditerranée et continent », dans K. Gruel \& T. Lejars (éd.), Établissements à vocation artisanale et réseaux d'échanges en Europe celtique entre Hallstatt et La Tène ( $8^{\mathrm{e}}$ journée internationale de Protohistoire celtique de l'ENS-Paris, 7 juin 2013), Paris. Disponible en ligne sur <www.archeo.ens.fr/spip.php?article566>.

UGOLINI Daniela, « The Greeks West of the Rhône (F): Genesis, Evolution and End of a Greek Area », Journal of Greek Archaeology, 3, 2018, p. 203-243.

UGOLINI Daniela, L'atelier de céramique grise monochrome du Mas de Pascal (Aspiran, Hérault, F). L'exemple d'une expérience ratée vers 500 av. J.-C., 2019. Disponible en ligne sur $<\mathrm{https} / / /$ hal.archivesouvertes.fr/hal-02066981/document>.

UGOLINI Daniela, «Les productions céramiques des Grecs du Midi : regards croisés ", dans M. Costanzi \& M. Dana (éd.), Une autre façon d'être grec : interactions et productions des Grecs en milieu colonial. - Another Way of Being Greek: Interactions and Cultural Innovations of the Greeks in a Colonial Milieu (Actes du colloque international d'Amiens, Paris, 2016), Louvain, Peeters, coll. « Colloquia Antiqua, 26 ", 2020, p. 407-432.

UGOLINI Daniela \& OLIVE Christian, « Un four de potier du ve s. av. J.-C. à Béziers, Place de la Madeleine », Gallia, 45, 1988, p. 13-28.

UGOLINI Daniela \& OLIVE Christian, « Sites grecs, sites indigènes. Essai sur le fonctionnement des habitats de l'Hérault occidental (vI $-\mathrm{IV}^{\mathrm{e}}$ s. av. J.-C.) », dans I. Bertrand, A. Duval, J. Gomez de Soto \& P. Maguer (éd.), Habitats et paysages ruraux en Gaule et regards sur d'autres régions du monde celtique (Actes du XXXI ${ }^{e}$ colloque international de l'AFEAF, Chauvigny, 17-20 mai 2007), Chauvigny, Mémoire XXXV de la Société archéologique champenoise, II, 2009, p. 215-243.

UGOLINI Daniela \& OLIVE Christian (éd.), Béziers I (600-300 av. J.-C.). La naissance de la ville, Béziers, Musée du Biterrois, 2006.

UGOLINI Daniela, OLIVE Christian, MARCHAND Georges \& COLUMEAU Philippe, « Un ensemble représentatif du v v s. av. J.-C. à Béziers, Place de la Madeleine, et essai de caractérisation du site ", DAM, 14, 1991, p. 141-203.

UGOLINI Daniela \& PARDIES Céline, «L'évolution topographique de l'habitat d'Agàthe (Agde, F) (vI ${ }^{\mathrm{e}}$ av. J.-C.-- $\mathrm{I}^{\mathrm{er}}$ s. apr. J.-C.) », Archeologia classica, LXIX, n. s. II.8, 2018, p. 167-196.

\section{NOTES}

1. Les dates s'entendent avant notre ère.

2. Ugolini et al. (1991).

3. Nous remercions très chaleureusement Raphaël Macario (HADES Archéologie, Toulouse), qui a autorisé l'utilisation de la documentation de l'îlot des Chaudronniers 2014; ainsi que le professeur Jean-Christophe Sourisseau (Aix-Marseille Université) et Lou de Barbarin (doctorante, Aix-Marseille Université) pour leur expertise des céramiques grecques de Saint-Jacques 2017-2018.

4. Pour la Sicile archaïque, F. de Polignac $(1999,225)$ évoque « des groupes hétérogènes dont le problème n'était pas seulement de trouver le site où s'établir, mais aussi de définir les contours de la collectivité qui s'y établirait ». 
5. Le terme « colonie » nous semble adapté aux caractéristiques de la ville en dépit de l'absence d'un récit de fondation et du nom de l'œciste dans les sources, des éléments censés justifier les colonies mais souvent bien postérieurs aux fondations.

6. Ugolini (2018) : nous y renvoyons pour compléter l'information sur les sources, la phase précoloniale et coloniale, la place de la ville dans le Midi, le mode de fonctionnement, le lien avec Agde, Rhòde ibérique.

7. Gomez, Ugolini \& Cros (2018) ; Ugolini (2018).

8. Seules quatre opérations d'une certaine ampleur ont eu lieu en plus de trente ans. L'addition des surfaces explorées, y compris par des sondages et suivis de travaux, est d'environ $6700 \mathrm{~m}^{2}$, soit environ $2 \%$ de la ville grecque. Pour comparaison, à Marseille, plus de $98000 \mathrm{~m}^{2}$ ont été explorés (d'après la Carte archéologique publiée en 2005 et augmentée depuis), soit environ $50 \%$ de la ville archaïque et $25-35 \%$ de la ville pré-hellénistique. Pourtant, beaucoup d'informations y font encore également défaut.

9. CAG 34/4, 144-148.

10. En dernier, Guilaine et al. (2017).

11. Gomez, Ugolini \& Cros (2018); Ugolini (2018).

12. CAG 34/4, s.v. Saint-Jacques.

13. Tréziny (2011a, 289, notamment fig. 2 : Mégara Hyblaea ; 292 et $n .13$ : Marseille).

14. D'après les mobiliers les plus récents des premiers comblements anthropiques du grand fossé (ST1) et de la citerne (ST84). Les analyses $14 \mathrm{C}$ ne contredisant pas cette proposition, voir BetaAnalytic, Rapport d'analyse de datation au radiocarbone, 10 décembre 2018, ST1-us $664: 696-540$ (67,7\% de probabilités), 642-556 (44,3\% de probabilités); ST1-us 685: 651-543 (45,7\% de probabilités) ; ST1-us 671 : 781-511 (95,4\% de probabilités), 648-546 (45,9\% de probabilités); ST84-us 965 : 696-540 (67,7 \% de probabilités), 642-556 (44,3\% de probabilités).

15. C'est, grosso modo, la taille estimée pour la Marseille contemporaine : cf. Tréziny (2011b, fig. 3).

16. Olive \& Ugolini (1997) ; Ugolini \& Olive (2006) ; CAG 34/4, s.v. Place de la Madeleine.

17. Ce n'est que plus tard ( $\mathrm{III}^{\mathrm{e}}$ siècle et plus largement $\mathrm{II}^{\mathrm{e}}$ siècle) que la maison « à cour » sera adoptée dans une certaine mesure par les autochtones. Lattes : voir Dietler et al. (2008); Pech Maho : voir Gailledrat et al. (2010, 265-267).

18. Macario $(2017,123-125)$.

19. Résultat de l'analyse de charbons de ce comblement par T.Goslar, dans Macario (2017, annexe 69 : 781-511 avec 95,4 \% de probabilités ; 648-546 avec 45,9\% de probabilités).

20. Hasaki (2002) ; Palmieri (2013, 59, n. 377 et fiches des sites). Olympie : voir Kunze, Schleif \& Eilmann (1939, fig. 22).

21. Comme le montrent les tablettes de Penteskouphia F871 et F639: cf. Hasaki (2002, pl. I, 6).

22. À Emporion, des excavations circulaires interprétées comme des fours de potiers sont datées à partir du second quart du $\mathrm{VI}^{\mathrm{e}}$ siècle : cf. Aquilué et al. (2001, 318-319).

23. Ugolini \& Olive (1988); CAG 34/4, s.v. Quartier La Madeleine.

24. CAG 34/4, s.v. Rue Mairan.

25. Macario (2017, 128, fig. 98-99).

26. Macario (2017, 129-131, pl. 20, fig. 108-122). L'analyse archéomagnétique a fixé la dernière montée en température entre 430 et 350 : cf. L. Garnier, dans Macario (2017, annexe 68).

27. Macario (2017, 133, fig. 100-104).

28. Macario (2017, 133, fig. 133-135).

29. Exemples dans Esposito \& Sanidas (2012).

30. Sur le corail, qui n'est pas extrait des côtes locales, et ses implications commerciales : Ugolini et al. (1991) ; Rondi-Costanzo (1997) ; Rondi-Costanzo \& Ugolini (2000) ; Ugolini (2006, 2013 et 2018).

31. Voir n. 16. 
32. Sur l'essai raté de l'atelier d'Aspiran/Mas de Pascal (Pezin, 2004), voir maintenant Ugolini (2019).

33. CAG 34/4, 109-124 ; Ugolini (2020).

34. CAG 34/4, 228-232; Macario (2017).

35. Travaux d'aménagement des eaux à Marseille : voir Tréziny (2009-2011, 50).

36. La documentation de ce sondage (par C. Lapeyre, conservateur du Musée du Biterrois), n'a pas été retrouvée : seuls quelques photos et croquis de situation sont disponibles, mais le mobilier est très abondant (en cours d'étude).

37. Pour celle connue de longue date, en dernier : Papaikonomou (2008, 697-705), qui développe le symbolisme funéraire de ces figurines. Nous remercions M. Santos (directrice du Museu d'Empùries) pour ses informations, notamment sur la poupée trouvée en 2010 dans une tombe inédite.

38. Roure et al. (2015).

39. P. ex., Calderone (1999, fig. 1 : Sélinonte) et La Rocca (2005: Crotone). En général : van der Meijden (1993).

40. Voir déjà Ugolini \& Olive (2009). Une mise à jour est en cours d'élaboration.

41. CAG 34/4, s.v. La Courondelle. Le Frigoulas : voir D. Gleizes et al., Béziers, ZAC du Frigoulas, Rapport 2013.

42. Lors de la publication par Olive, Ugolini \& Ratsimba (2009), le four n'avait pas pu être précisément daté d'après le mobilier. L'analyse archéomagnétique de la structure a fixé la dernière montée en température en 368 , avec $95 \%$ de confiance : cf. G. Hervé \& P. Lanos, Béziers (Hérault), ZAC de la Domitienne, four à pithoi. Analyse archéomagnétique, Rennes, 15 mai 2009.

43. P. ex., aux Halles 1986: 601 bords d'amphores de la phase grecque (600-300) dont 258 massaliètes, 148 étrusques, 111 ibériques, 76 grecques diverses, 8 puniques.

44. Aucune ferme ne semble avoir été découverte ou localisée à la périphérie de Marseille, mais des vignes : en dernier, Bouffier \& Garcia (2014). Pour Emporion, R. Plana-Mallart, dans MartinezSève (2012, 157-178), suppose que les relations économiques entre autochtones et colons ont stimulé l'apparition du hameau de Pontos et des quelques fermes indigènes localisées à quelque distance de l'établissement grec. Des estimations très différentes ont concerné la chôra d'Agde jusqu'aux recherches de Gomez (2010), qui ont prouvé la mise en place d'un territoire uniquement après 150 , c'est-à-dire lors de la phase massaliète (Agde II), et principalement sur la rive gauche de l'Hérault.

45. Sur les problèmes d'appréciation des premières implantations grecques en Italie, de la diversité des cas, du passage de l'empòrion à l'apoikìa et du rôle des indigènes, voir Esposito (2012). Béziers I/Rhòde n'échappe évidemment pas à ces difficultés.

46. Sur Agde et ses phases, en dernier Ugolini \& Pardies (2018); sur les conséquences de la création de ce port dans les rapports avec les indigènes, voir Ugolini (2018).

47. La Pyréne des textes - liée à Héraclès (Silius Italicus, Punica, III, 415-440), figure majeure pour les Doriens - était autrefois située à Port-Vendres. Après une allusion indirecte de Ponsich $(1975,10)$, sa localisation à Collioure a été proposée : cf. Ugolini $(1998,82-83)$ et Ugolini $(2005,3)$. Son port, fréquenté par les Grecs (Avienus, Ora Maritima, v. 558-561), encore en fonction au début du II ${ }^{\mathrm{e}}$ siècle (Tite-Live, XXXIV, 34, 8), est maintenant assuré : en dernier, Dunyach (2018, I, 66-115 et III, 9-64). Dans la mention d'Hérodote II, 33, Pyréne semble associée à l'extrême Occident tout en étant située aux sources de l'Istros (Danube). L'hypothèse qu'il s'agisse du site hallstattien de la Heuneburg (au bord du Danube, à $80 \mathrm{~km}$ de sa source) est maintenant mise en avant: en dernier, Fernandez-Götz (2015).

48. Ugolini (2018). 


\section{RÉSUMÉS}

La phase grecque de Béziers (Béziers I) a été identifiée seulement depuis quelques décennies et la ville est restée longtemps anonyme. Des opérations préventives récentes ont mis au jour des vestiges de la période archaïque qui ont renouvelé les problématiques. Le site a été investi avant 600 av. J.-C., c'est-à-dire avant Marseille, dans le cadre d'un mouvement dorien/rhodien qui a précédé celui ionien/phocéen et qui ressort aussi par l'ensemble du dossier concernant la deuxième moitié $\mathrm{du} \mathrm{VII}^{\mathrm{e}}$ siècle en Languedoc. Grâce à ces nouveaux acquis, le nom de Rhòde, consigné dans les sources, a pu lui être attribué. Sont présentées ici les nouvelles données de l'archéologie locale et le développement de la ville, désormais considérée comme une polis, jusqu'à son abandon vers 300 av. J.-C.

The Greek phase of Béziers (Béziers I) was identified only a few decades ago and the city remained anonymous for a long time. Recent excavations uncovered remains of the archaic period that have renewed the historical context. The site was occupied before $600 \mathrm{BC}$, that is before Marseille, by a Dorian/Rhodian movement-preceding the Ionian/Phocaean one-and also emerging from the data concerning the second half of the 7th century in the Languedoc. Thanks to this new evidence, the name of Rhòde, recorded in the sources, could be attributed to Béziers I. Here are presented the new data of local archaeology and the development of the city, now considered a polis, until its abandonment around $300 \mathrm{BC}$.

\section{INDEX}

Mots-clés : artisanat, colonisation, fortification, habitat, pratiques religieuses, potiers Keywords : crafts, colonization, fortification, habitat, religious practices, potters

\section{AUTEURS}

\section{ÉLIAN GOMEZ}

Service archéologique municipal de Béziers

Chercheur associé à l'UMR 7299 - Centre Camille Jullian,

Aix-Marseille Université - CNRS - Ministère de la Culture, Aix-en-Provence

elian.gomez@ville-beziers.fr

\section{DANIELA UGOLINI}

Aix-Marseille Université - CNRS - Ministère de la Culture, Aix-en-Provence UMR 7299 - Centre Camille Jullian dugolini@club-internet.fr 